\title{
Periphytic and planktonic algae records from the upper Paraná river floodplain, Brazil: an update
}

\author{
- D. Bárbara Dunck ${ }^{1,4}$, Maria Gabriela Junqueira ${ }^{2}$, Andressa Bichoff ${ }^{3}$, Matheus Vieira da Silva ${ }^{2}$, \\ Alfonso Pineda ${ }^{3}$, Aline Caroline Magro de Paula ${ }^{3}$, Bárbara Furigo Zanco ${ }^{3}$, \\ Geovani Arnhold Moresco ${ }^{3}$, Patrícia Iatskiu ${ }^{3}$, Jascieli Carla Bortolini ${ }^{3}$, Yasmin Rodrigues de Souza ${ }^{3}$, \\ Sueli Train ${ }^{3}$, Luzia Cleide Rodrigues ${ }^{3}$, Susicley Jati ${ }^{3}$ and Liliana Rodrigues ${ }^{3}$
}

Received: 11.01.2018; accepted: 02.08.2018

\begin{abstract}
Periphytic and planktonic algae records from the upper Paraná river floodplain, Brazil: an update). The upper Paraná river floodplain is a strategic area for the Brazilian biological and environmental patrimony and contains several conservation units. We aimed to record the occurrence and the geographic distribution of microalgae in this floodplain during 30 years of research carried out by the Center for Research in Limnology, Ichthyology and Aquaculture - State University of Maringá. We consulted 80 publications (national and international journals and books published from 1986 to 2016). We considered only published works that referenced algae at the generic and infrageneric levels. The results indicated 938 species of algae (562 periphytic, 482 planktonic), which 103 co-occur in the two habitats. Cosmarium was the richest genera for periphyton and Traquelomonas for phytoplankton. This study increased the knowledge of microalgae biodiversity in Brazil and provided data for future ecological and biogeographic studies.
\end{abstract}

Keywords: freshwater, inventory, microalgae, phycologia, phytoplankton

RESUMO - (Registros de algas perifíticas e planctônicas da planície de inundação do alto rio Paraná, Brasil: uma atualização). A planície de inundação do alto rio Paraná é uma área estratégica para o patrimônio biológico brasileiro por conter várias unidades de conservação. Objetivamos registrar a ocorrência e a distribuição geográfica das microalgas ocorrentes nesta planície durante 30 anos de pesquisas realizadas na região pelo Núcleo de Pesquisas em Limnologia, Ictiologia e Aquicultura da Universidade Estadual de Maringá. Foram consultadas 80 publicações (periódicos nacionais e internacionais, e livros publicados desde 1994 até 2016), das quais foram consideradas apenas publicações que referenciam algas no nível genérico e infragenérico. Os resultados indicaram 938 espécies de algas (562 perifíticas e 482 planctônicas), das quais 103 coocorrem nos dois habitats. Cosmarium foi o gênero com maior número de espécies no perifíton e Traquelomonas para o fitoplâncton. Este estudo ampliou o conhecimento da biodiversidade de microalgas no Brasil e forneceu dados para futuros estudos ecológicos e biogeográficos.

Palavras-chave: água doce, ficoperifíton, fitoplâncton, inventário, microalgas

\section{Introduction}

Regarding the flora of aquatic organisms, algae are important contributors to overall primary productivity. They comprise a polyphyletic grouping of both prokaryotic and eukaryotic producers (autotrophic or heterotrophic protists), important on the trophic webs to act on primary productivity and as food for various herbivores. They can be defined as single-celled organisms, which do not have reproductive organs involved by sterile cell layers, with a wide variety of forms, functions and strategies (Bicudo \& Menezes 2010).

Brazil has an extensive hydrographic network, rich in natural rivers, lagoons and canals, and yet, proportionally, there is scarce knowledge about

1. Universidade Federal do Pará, Programa de Pós-graduação em Ecologia, Rua Augusto Corrêa, 1, Campus Guamá, 66075-110 Belém, PA, Brasil

2. Universidade Estadual de Maringá, Departamento de Biologia, Av. Colombo, 5790, 87020-900 Maringá, PR, Brasil

3. Universidade Estadual de Maringá, Programa de Pós-Graduação em Ecologia de Ambientes Aquáticos Continentais, Av. Colombo, 5790, 87020-900 Maringá, PR, Brasil

4. Corresponding author: dunck.barbara@gmail.com 
the biodiversity and the dynamics of microalgae (Agostinho et al. 2004, Menezes et al. 2015). The continental dimensions of this country, the high diversity, lack of laboratory facilities and the high logistic cost make the records of occurrence restricted to the geographic areas of activity of the few research groups (Bicudo \& Menezes 2010, Grieneisen et al. 2014, Menezes et al. 2015). Until now, the species distribution among the Brazilian states and the geopolitical regions are considered heterogeneous with predominance in southeast and south regions (Menezes et al. 2015).

Some surveys have been carried out to demonstrate the documented flora of these microorganisms in Brazil. Currently, Menezes et al. (2015) have compiled data to update the list of algae and cyanobacteria from Brazil previously published by Bicudo \& Menezes (2010) in the Catalog of Plants and Fungi from Brazil (Forzza et al. 2010). This update showed that there are 4,747 species registered in different environments and biomes in Brazil. However, the same update also indicated that this estimate is far from the actual algal diversity present in Brazil, due to the extensive geographical area represented by this country and the gaps in scientific research.

The Brazilian southern region houses one of the main floodplains of the country, the upper Paraná river floodplain $\left(54^{\circ} 20^{\prime}-53^{\circ} 30^{\prime} / 24^{\circ} 00^{\prime}-22^{\circ} 50^{\prime}\right)$. It is located on the right margin of the last stretch of this river that is free of reservoirs in the Brazilian territory, located between the reservoirs of Porto Primavera and Itaipu. This region has a recognized strategic area for the Brazilian biological and environmental patrimony, with two federal conservation units (Ilha Grande National Park and APA of the Islands and Várzeas of the Paraná River) and one state park (Ivinheima State Park). The upper Paraná river floodplain was incorporated into the Biosphere Reserve of the Atlantic Forest-t MAB/UNESCO (Agostinho et al. 2004, Takemoto \& Lizama 2010).

Studies in this region have been developed since 1986 by researchers from Nupélia (Center for Research in Limnology, Ichthyology and Aquaculture - State University of Maringá), who are responsible for one of the most important databases on Freshwater biology and ecology in Latin America. Plenty of information about the characteristics of the environment, fauna and flora is contained in scientific papers, technical reports (available at http://www.peld.uem.br/), and papers of diffusion, theses and dissertations of the Program of Postgraduate in Ecology of Continental
Aquatic Environments of the State University of Maringá. Some researches have indicated that the freshwater microalgae of this area represent $8 \%$ of the total microalgae found in Brazilian territory (Train \& Rodrigues 2004, Agostinho et al. 2004). Indeed, despite the number of already developed taxonomic and ecological, observational and/or experimental studies, there are still background gaps in the biodiversity of microalgae in this area. In this way, this work aimed to record and disseminate information of the microalgae (periphytic and planktonic) occurring in the upper Paraná river floodplain based on scientific papers published since 1986 .

\section{Materials and methods}

Study area - The study area covers a variety of lakes with different degrees of connectivity with the Paraná River and its main tributaries (Ivinhema River and Baia River) in the upper Paraná river floodplain (54²0'$53^{\circ} 30^{\prime} / 24^{\circ} 00^{\prime}-22^{\circ} 50^{\prime}$, figure 1$)$. The researches used in this survey include three rivers (Paraná, Ivinhema and Baia) and 20 lakes (six associated to the Paraná RiverGarças, Pombas, Osmar, Clara, Carioca and Pousada; seven to Baía River- Guaraná, Maria Luiza, Porcos, Fechada, Aurélio, Pousada das Garças and Gavião; seven to Ivinheima River- Patos, Finado Raimundo, Ipoitã, Capivara, Jacaré, Peroba and Ventura).

Review of articles - To create a catalog of species and respective articles in which they are cited we use papers and books published nationally and internationally. We consult the basis of Scielo, Web of Science, Lattes platform, the library of the Program

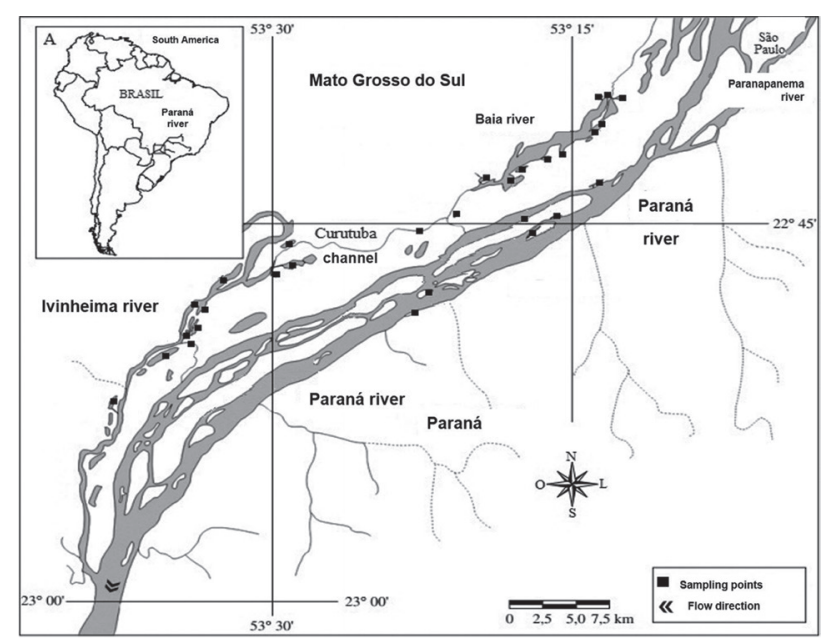

Figure 1. The upper Paraná River floodplain and environments sampled over the years until 2006. 
of Postgraduate in Ecology of Continental Aquatic Environments of the State University of Maringá and Nupelia, and teachers' personal collection to do this survey.

As the goal was to look for scientific papers published since 1986, we noted that the first published works date of the year 1994. Thus, we use data from 1994 to 2016 . We considered only works that referenced algae at the generic and infrageneric level (excluding studies referring only to large groups or algal biomass). Species richness was considered as the sum of specific and infraspecific taxonomic units (even if cited for more than one environment) recorded in the floodplain. We consider also the number of species registers counting the number of studies in which they are registered.

The species are presented and organized by classes, both species and classes in alphabetical order. The classification system adopted was Round (1971) for all algae classes. The species in the catalog are presented with the respective articles in which they were cited. We present the updated species name based on Algaebase (http://www.algaebase.org/) including as synonymous $(=)$ the species name cited in each work we consulted. We also included species cited as conferatum, and the variety of species when were cited in the papers.

\section{Results and Discussion}

From the 80 national and international papers published in scientific journals and book chapters (taxonomic studies, ecological, experimental and observational studies) published from 1994 to 2016, we used 41 because lots of them only referring to large algae groups or algal biomass. These works encompassed 28 papers of periphytic algae and 13 of planktonic algae. The scientific published studies used are shown in table 1.

The results of the survey indicated 938 species of algae occurring in the upper Paraná river floodplain, of which 103 co-occur in both periphytic and planktonic habitats (table 2). The Chlorophyceae class was the richest one (199 taxa) followed by Zygnemaphyceae (196) (table 2). In the periphytic habitat Bacillariophyceae, Cyanophyceae and Zygnemaphyceae (figure 2) were predominant, and Chlorophyceae and Euglenophyceae in phytoplankton (figure 2).

Periphyton - The upper Paraná river floodplain of presented 562 taxa of periphytic algae (table
2) distributed into 10 classes (Bacillariophyceae, Cyanophyceae, Chlorophyceae, Chrysophyceae, Euglenophyceae, Cryptophyceae, Oedogoniophyceae, Rhodophyceae, Zygnemaphyceae and Xanthophyceae). Bacillariophyceae was the richest class (150 taxa) followed by Cyanophyceae (149 taxa) and Zygnemaphyceae (128 taxa). The richest genera were Cosmarium (40 species) followed by Eunotia (22 species) and Staurastrum (22 species) (table 2).

The species with the largest number of registers were Achnanthidium minutissimun Kützing (12 researchs), Fragilaria capucina Desmazières (11 researchs), Gomphonema gracile Ehrenberg (11 researchs), Gomphonema parvulum (Kützing) Kützing (10 researchs), Leptolyngbya perelegans (Lemmermann) Anagnostidis \& Komárek = Lyngbya perelegans Lemmermann (10 researchs) and Ulnaria ulna (Nitzsch) Compère (nine researchs) (table 2).

Phytoplankton - The upper Paraná river floodplain presented 482 species of planktonic algae distributed into eight classes (Bacillariophyceae, Cyanophyceae, Chlorophyceae, Chrysophyceae, Euglenophyceae, Cryptophyceae, Zygnemaphyceae and Xanthophyceae). Chlorophyceae was the richest class (148) followed by Euglenophyceae (102) and Zygnemaphyceae (98). The richest genus was Traquelomonas (60), followed by Staurastrum (25) and Phacus (22) (table 2).

The species with the largest number of registers were Aulacoseira granulata var. granulata (Ehrenberg) Simonsen, Dolichospermum circinalis (Rabenhorst ex Bornet \& Flahault) Wacklin, Hoffmann \& Komárek (Anabaena circinalis Rabenhorst ex Bornet \& Flahault) and Trachelomonas volvocinopsis Swirenko in six

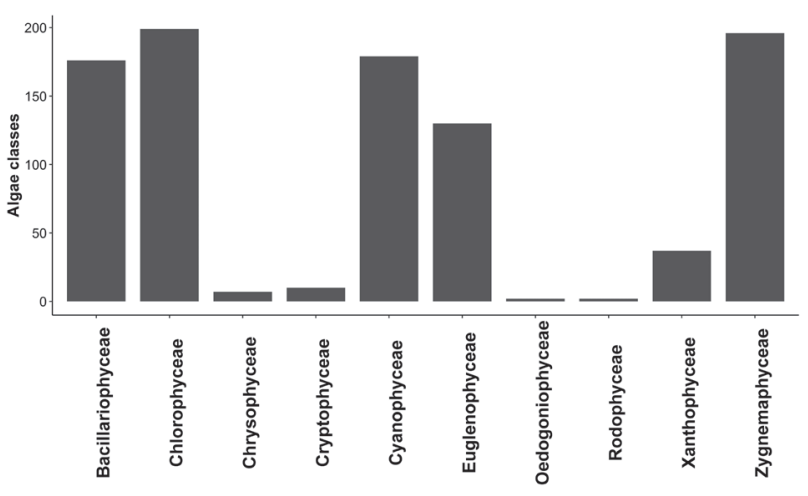

Figure 2. Proportion among taxonomic classes in the upper Paraná River floodplain. 
Table 1. Periphytic and planktonic microalgae studies used in the compilation of taxa occurring in the floodplain from 1994 to 2016 (1-28: Periphytic habitat, in alphabetical order; 29-41: Planktonic habitat, in alphabetical order).

\begin{tabular}{|c|c|c|}
\hline Reference & Citation & Habitat \\
\hline 1 & Algarte \& Rodrigues (2013) & Periphyton \\
\hline 2 & Algarte et al. (2006) & Periphyton \\
\hline 3 & Algarte et al. (2009) & Periphyton \\
\hline 4 & Algarte et al. (2014) & Periphyton \\
\hline 5 & Algarte et al. (2015) & Periphyton \\
\hline 6 & Algarte et al. (2016) & Periphyton \\
\hline 7 & Bichoff et al. (2016) & Periphyton \\
\hline 8 & Biolo \& Rodrigues (2010) & Periphyton \\
\hline 9 & Biolo \& Rodrigues (2011) & Periphyton \\
\hline 10 & Biolo \& Rodrigues (2013a) & Periphyton \\
\hline 11 & Biolo \& Rodrigues (2013b) & Periphyton \\
\hline 12 & Biolo et al. (2015) & Periphyton \\
\hline 13 & Cabral et al. (2014) & Periphyton \\
\hline 14 & Dunck et al. (2013) & Periphyton / Phytoplankton \\
\hline 15 & Dunck et al. (2015) & Periphyton \\
\hline 16 & Dunck et al. (2016a) & Periphyton \\
\hline 17 & Dunck et al. (2016b) & Periphyton \\
\hline 18 & Ferreira et al. (2011) & Periphyton \\
\hline 19 & Fonseca \& Rodrigues (2004) & Periphyton \\
\hline 20 & Fonseca \& Rodrigues (2005) & Periphyton \\
\hline 21 & Fonseca \& Rodrigues (2007) & Periphyton \\
\hline 22 & Mormul et al. (2010) & Periphyton \\
\hline 23 & Murakami \& Rodrigues (2009) & Periphyton \\
\hline 24 & Neif et al. (2013) & Periphyton \\
\hline 25 & Neif et al. (2014) & Periphyton \\
\hline 26 & Osório et al. (2016) & Periphyton \\
\hline 27 & Rodrigues \& Bicudo (2001) & Periphyton \\
\hline 28 & Rodrigues \& Bicudo (2004) & Periphyton \\
\hline 29 & Bortolini et al. (2014) & Phytoplankton \\
\hline 30 & Bortolini et al. (2016) & Phytoplankton \\
\hline 31 & Bovo-Scomparin et al. (2005) & Phytoplankton \\
\hline 32 & Bovo-Scomparin et al. (2013) & Phytoplankton \\
\hline 33 & Jati \& Tain (1993) & Phytoplankton \\
\hline 34 & Jati \& Train (1994) & Phytoplankton \\
\hline 35 & Moresco et al. (2015) & Phytoplankton \\
\hline 36 & Oliveira et al. (1994) & Phytoplankton \\
\hline 37 & Paula et al. (2014) & Phytoplankton \\
\hline 38 & Rodrigues et al. (2009) & Phytoplankton \\
\hline 39 & Train \& Rodrigues (1998) & Phytoplankton \\
\hline 40 & Train et al. (2004) & Phytoplankton \\
\hline 41 & Train et al. (2000) & Phytoplankton \\
\hline
\end{tabular}


Table 2. Occurrence of periphytic and planktonic algae in the upper Paraná river floodplain and respective citations. The updated species name was based on Algaebase; equal symbol $(=)$ indicates the species synonymous (heterotypic, taxonomic synonym) cited in the consulted work, and ( $\equiv$ ) indicates the homotypic synonym (if cited in the work consulted); numerals (1 to 41) indicate the citation reference and habitat (1-28: periphytic habitat; 29-41: planktonic habitat) of the list in Table 1 ; asterisk (*) indicates the species common to both habitats.

\begin{tabular}{|c|c|}
\hline Family/Species & Reference \\
\hline \multicolumn{2}{|l|}{ Bacillariophyceae } \\
\hline Acanthoceras magdeburgense Hongimann & 40 \\
\hline Achnanthes inflata (Kützing) Grunow & 27 \\
\hline \multicolumn{2}{|l|}{ Achnanthes lanceolata (Brébisson) Grunow } \\
\hline 三Planothidium lanceolatum (Brébisson ex Kützing) Lange-Bertalot & 18,27 \\
\hline Achnanthes rupestoides Hohn & 18 \\
\hline \multicolumn{2}{|l|}{ Achnanthidium exiguum (Grunow) Czarnecki } \\
\hline$\equiv$ Achnanthes exigua Grunow & $2,18,27$ \\
\hline Achnanthidium minutissimum (Kützing) Czarnecki & $\begin{array}{c}1,2,6,7,9,10,12,14,16,18,22,23,24,27 \\
28^{*}\end{array}$ \\
\hline$\equiv$ Achnantes minutissima Kützing & 40 \\
\hline Amphipleura lindheimeri Grunow & $7,18,27,29,40^{*}$ \\
\hline Amphora copulata (Kützing) Schoeman \& Archibald & 16,18 \\
\hline Aulacoseira agassizii (Ostenfeld) Simonsen & 39,40 \\
\hline Aulacoseira alpigena (Grunow) Krammer & 16,18 \\
\hline Aulacoseira ambígua (Grunow) Simonsen & $2,18,27$ \\
\hline Aulacoseira ambigua var. ambigua (Grunow) Simonsen & $29,34,39,40$ \\
\hline Aulacoseira ambigua var. ambigua f. spiralis (Skuja) Ludwig & 40 \\
\hline Aulacoseira distans (Ehrenberg) Simonsen & $16,27,29,34,39,30,40^{*}$ \\
\hline Aulacoseira granulata (Ehrenberg) Simonsen & $2,7,16,18,23,27,28$ \\
\hline Aulacoseira granulata (Ehrenberg) Simonsen var. granulata & $29,30,34,38,39,40$ \\
\hline Aulacoseira granulata var. angustissima (Müller) Simonsen & $16,39,40^{*}$ \\
\hline $\begin{array}{l}\text { Aulacoseira granulata (Ehrenberg) Simonsen var. angustissima } \\
\text { (Müller) Simonsen f. curvata Grunow }\end{array}$ & 40,41 \\
\hline $\begin{array}{l}\text { Aulacoseira granulata (Ehrenberg) Simonsen var. valida (Hustedt) } \\
\text { Simonsen }\end{array}$ & 16 \\
\hline Aulacoseira herzogii (Lemmermann) Simonsen & $15,27,40,30^{*}$ \\
\hline Aulacoseira muzzanensis (Meister) Krammer & $16,29 *$ \\
\hline Aulacoseira pseudogranulata (Cleve-Euler) Simonsen & 40 \\
\hline \multicolumn{2}{|l|}{ Brachysira vitrea (Grunow) Ross } \\
\hline$\equiv$ Anomoeoneis vitrea (Grunow) Ross & 23,27 \\
\hline Cocconeis pediculus Ehrenberg & 22 \\
\hline Cocconeis placentula Ehrenberg & $9,16,18,22,24,27$ \\
\hline \multicolumn{2}{|l|}{ Craticula cuspidata (Kutzing) Mann } \\
\hline$\equiv$ Navicula cuspidata (Kutzing) Kutzing & 27 \\
\hline Cyclotella meneghiniana Kützing & $2,18,23,27,40,41^{*}$ \\
\hline Cymbella affinis Kützing & $12,18,23,27,40,41^{*}$ \\
\hline Cymbella gracilis (Ehrenberg) Kützing & $1,12,23,27$ \\
\hline Cymbella tumida (Brébisson) Van Heurck & 2,27 \\
\hline
\end{tabular}


Table 2 (continuation)

\begin{tabular}{|c|c|}
\hline Family/Species & Reference \\
\hline Cymbopleura anglica (Lagerstedt) Krammer: & 16 \\
\hline Diadesmis confervacea Kützing: & 18 \\
\hline Diploneis elliptica (Kützing) Cleve: & 27 \\
\hline Diploneis ovalis (Nielse) Cleve: & 40 \\
\hline \multicolumn{2}{|l|}{ Diploneis subovalis Cleve: 2, 27} \\
\hline Discostella stelligera (Cleve \& Grunow) Holk \& Klee & $16,38,40$ \\
\hline$\equiv$ Cyclotella stelligera (Cleve \& Grunow) Van Heurck & $22,27,30,39,41 *$ \\
\hline Encyonema angustecapitatum Krammer & 16 \\
\hline Encyonema mesianum (Cholnoky) Mann & $1,2,9,10,12,24$ \\
\hline$\equiv$ Cymbella mesiana Cholnoky & 27 \\
\hline Encyonema minutum (Hilse) Mann & $1,2,6,18,22,23,23,40^{*}$ \\
\hline$\equiv$ Cymbella minuta Hilse & 27,28 \\
\hline Encyonema neogracile Krammer & 16 \\
\hline Encyonema neomesianum Krammer & 7,16 \\
\hline Encyonema perpusillum (Cleve-Euler) Mann & 18 \\
\hline Encyonema silesiacum (Bleisch) Mann & $1,2,6,7,9,10,16,18,22,23,24,40^{*}$ \\
\hline$\equiv$ Cymbella silesiaca Bleisch & 27 \\
\hline Eunotia bilunaris (Ehrenberg) Schaarschmidt & $16,18,23,27$ \\
\hline$=$ Eunotia curvata (Kützing) Lagerstedt & 2 \\
\hline Eunotia camelus Ehrenberg & $16,23,27,39,40^{*}$ \\
\hline Eunotia cf. serra Ehrenberg & 2 \\
\hline Eunotia didyma Grunow & 2,16 \\
\hline Eunotia didyma var. didyma Grunow & 40 \\
\hline \multicolumn{2}{|l|}{ Eunotia excelsa (Krasske) Nörpel } \\
\hline$=$ Eunotia rabenhorstii Cleve et Grunow & 2 \\
\hline Eunotia faba (Ehrenberg) Grunow & 10 \\
\hline Eunotia flexuosa (Brébisson ex Kützing) Kützing & $2,9,16,23,24,27,40^{*}$ \\
\hline Eunotia incisa Smith ex Gregory & $16,23,27$ \\
\hline Eunotia indica Grunow & 6 \\
\hline Eunotia intermedia (Krasske ex Hustedt) Nörpel \& Lange-Bertalot & 10,12 \\
\hline Eunotia lineolata Hustedt & $2,23,27,40^{*}$ \\
\hline Eunotia major (Smith) Rabenhorst & 10 \\
\hline Eunotia minor (Kützing) Grunow & $9,18,22,23,24,27$ \\
\hline Eunotia monodon Ehrenberg & $18,39,40^{*}$ \\
\hline Eunotia neomundana Metzeltin \& Lange-Bertalot & 10 \\
\hline Eunotia nymanniana Grunow & 2 \\
\hline Eunotia pectinalis (Kützing) Rabenhorst & $10,18,23,27$ \\
\hline Eunotia praerupta Ehrenberg & $18,23,27$ \\
\hline Eunotia rabenhorstiana (Grunow) Hustedt & 16 \\
\hline Eunotia sudetica Müller: & $2,7,23,27$ \\
\hline Eunotia zygodon Ehrenberg & 16 \\
\hline Fragilaria capucina Desmazières & $2,6,7,70,10,11,14,23,24,25,27,28,39,40^{*}$ \\
\hline
\end{tabular}


Table 2 (continuation)

\begin{tabular}{|c|c|}
\hline Family/Species & Reference \\
\hline Fragilaria goulardii (Brébisson ex Grunow) Lange-Bertalot & 27 \\
\hline = Synedra goulardii Brébisson ex Cleve \& Grunow & $6,7,22,25$ \\
\hline Fragilaria gracilis Østrup & 14 \\
\hline Fragilaria rumpens (Kützing) Carlson & 22 \\
\hline Fragilaria tenera (Smith) Lange-Bertalot & $10,11,25$ \\
\hline Fragilaria ulna (Nitzsch) Lange-Bertalot & 27 \\
\hline \multicolumn{2}{|l|}{ Fragilariforma javanica (Hustedt) Wetzel, Morales \& Ector } \\
\hline$\equiv$ Fragilaria javanica Hustedt: & 14 \\
\hline Frustulia cf. zizkae Lange-Bertalot & 6 \\
\hline Frustulia pumilio Lange-Bertalot \& Rumrich & 7 \\
\hline Frustulia rhomboides (Ehrenberg) De Toni & $2,23,25,27,40,41^{*}$ \\
\hline Frustulia saxonica Rabenhorst: & 7,14 \\
\hline Frustulia vulgaris (Thwaites) Toni & 40 \\
\hline \multicolumn{2}{|l|}{ Gomphoneis clevei (Fricke) Gil } \\
\hline$\equiv$ Gomphonema clevei Fricke & $10,23,25$ \\
\hline Gomphonema affine Kützing & $2,23,24,25,26,27$ \\
\hline Gomphonema angustatum (Kützing) Rabenhorst & 26 \\
\hline Gomphonema angustum Agardh & 22,27 \\
\hline Gomphonema augur Ehrenberg & $2,7,14,23,25,27,40,41^{*}$ \\
\hline Gomphonema brasiliense Grunow & $1,2,7,10,11,23,25,26$ \\
\hline Gomphonema costei Metzeltin \& Lange-Bertalot & 26 \\
\hline Gomphonema gracile Ehrenberg & $1,2,6,7,10,11,14,22,23,24,25,27,40^{*}$ \\
\hline Gomphonema insigne Gregory & 7 \\
\hline Gomphonema lagenula Kützing & 26 \\
\hline Gomphonema laticollum Reichardt & 26 \\
\hline \multicolumn{2}{|l|}{$\begin{array}{l}\text { Gomphonema subclavatum (Grunow) Grunow var. mexicanum } \\
\text { (Grunow) Patrick }\end{array}$} \\
\hline$=$ Gomphonema mexicanum Grunow & 7,26 \\
\hline Gomphonema neonasutum Lange-Bertalot \& Reichardt & 26 \\
\hline Gomphonema parvulum (Kützing) Kützing: & $2,6,7,10,14,22,23,25,26,27,28,40^{*}$ \\
\hline Gomphonema pseudoaugur Lange-Bertalot & 2 \\
\hline Gomphonema pumilum (Grunow) Reichardt \& Lange-Bertalot & $7,24,26$ \\
\hline Gomphonema salae Lange-Bertalot \& Reichardt & 26 \\
\hline Gomphonema sphaerophorum Ehrenberg & 26 \\
\hline Gomphonema subtile Ehrenberg & $1,2,14,23,26,27,40^{*}$ \\
\hline Gomphonema truncatum Ehrenberg & $10,23,25,27,40^{*}$ \\
\hline Gomphonema turris Ehrenberg: & 7,14 \\
\hline Gomphonema turris Ehrenberg var. coarctata (Frenguelli) Frenguelli & 26 \\
\hline Gyrosigma cf. spencerii (Smith) Griffith \& Henfrey & 40 \\
\hline \multicolumn{2}{|l|}{ Gyrosigma sciotense (Sullivan \& Wormley) Cleve } \\
\hline$=$ Gyrosigma nodiferum (Grunow) Reimer & 27 \\
\hline Gyrosigma scalproides (Rabenhorst) Cleve & 25 \\
\hline
\end{tabular}


Table 2 (continuation)

\begin{tabular}{|c|c|}
\hline Family/Species & Reference \\
\hline Hantzschia amphioxys (Ehrenberg) Grunow & $27,40^{*}$ \\
\hline \multicolumn{2}{|l|}{$\begin{array}{l}\text { Hippodonta capitata (Ehrenberg) Lange-Bertalot, Metzeltin \& } \\
\text { Witkowski }\end{array}$} \\
\hline$\equiv$ Navicula capitata Ehrenberg & 27 \\
\hline \multicolumn{2}{|l|}{$\begin{array}{l}\text { Humidophila contenta (Grunow) Lowe, Kociolek, Johansen, } \\
\text { Van de Vijver, Lange-Bertalot \& Kopalová }\end{array}$} \\
\hline$\equiv$ Navicula contenta Grunow & 27 \\
\hline Hydrosera whampoensis (Schwarz) Deby & 24 \\
\hline \multicolumn{2}{|l|}{ Iconella delicatissima (Lewis) Ruck \& Nakov } \\
\hline$\equiv$ Stenopterobia delicatissima (Lewis) Brébisson ex Van Heurck & 14 \\
\hline \multicolumn{2}{|l|}{ Iconella linearis (Smith) Ruck \& Nakov } \\
\hline$\equiv$ Surirella linearis Smith & 24,27 \\
\hline \multicolumn{2}{|l|}{ Iconella tenera (Gregory) Ruck \& Nakov } \\
\hline$\equiv$ Surirella tenera Gregory & 14,27 \\
\hline \multicolumn{2}{|l|}{ Luticola mutica (Kützing) Mann } \\
\hline$\equiv$ Navicula mutica Kützing & 27 \\
\hline Melosira varians Agardh & $2,7,14,25,27,28,40^{*}$ \\
\hline Navicula cf. capitatoradiata Germain & 25 \\
\hline Navicula cf. cryptocephala Kützing & 23 \\
\hline Navicula cf. reinhardtii (Grunow) Grunow & 40 \\
\hline Navicula cf. trivialis Lange-Bertalot & 10 \\
\hline Navicula cryptocephala Kützing & $2,6,14,23,24,27,40^{*}$ \\
\hline Navicula cryptotenella Lange-Bertalot & $1,7,25$ \\
\hline Navicula halophila (Grunow) Cleve & 40 \\
\hline Navicula radiosa Kützing & 27 \\
\hline Navicula schroeteri Meister & $25,27,40^{*}$ \\
\hline Navicula viridula (Kützing) Ehrenberg & $23,25,27$ \\
\hline Neidium affine (Ehrenberg) Pfitzer & 37 \\
\hline Neidium iridis (Ehrenberg) Cleve & 40 \\
\hline Nitzschia acicularis (Kützing) Smith & $27,39,40^{*}$ \\
\hline Nitzschia amphibia Grunow & $2,6,10,23,24,25,27$ \\
\hline Nitzschia clausii Hantzsch: & 7,27 \\
\hline Nitzschia filiformis (Smith) Van Heurck & 40 \\
\hline Nitzschia gracilis Hantzsch & 39,40 \\
\hline Nitzschia ignorata Krasske & 2,25 \\
\hline Nitzschia linearis Smith & $10,11,24,25$ \\
\hline Nitzschia palea (Kützing) Smith & $2,7,10,11,14,23,25,27,28,39,40,41 *$ \\
\hline Nitzschia sigma (Kützing) Smith & 27 \\
\hline Nitzschia sigmoidea (Nitzsch) Smith & 22 \\
\hline \multicolumn{2}{|l|}{ Pantocsekiella kuetzingiana (Thwaites) Kiss \& Ács } \\
\hline$\equiv$ Cyclotella kuetzingiana Thwaites & 27 \\
\hline Pinnularia acrosphaeria Smith: & $6,14,27,41$ \\
\hline
\end{tabular}


Table 2 (continuation)

\begin{tabular}{|c|c|}
\hline Family/Species & Reference \\
\hline Pinnularia cf. doeringii (Frenguelli) Mills & 24 \\
\hline Pinnularia cf. gibba Ehrenberg & 25 \\
\hline Pinnularia cf. microstauron (Ehrenberg) Cleve & 40 \\
\hline \multicolumn{2}{|l|}{ Pinnularia brauniana (Grunow) Studnicka } \\
\hline$=$ Pinnularia braunii (Grunow) Cleve & $23,27,41^{*}$ \\
\hline Pinnularia divergens Smith & 27 \\
\hline Pinnularia gibba Ehrenberg: & $27,40,41^{*}$ \\
\hline Pinnularia latarea Krammer & 7 \\
\hline Pinnularia major (Kützing) Rabenhorst & 40 \\
\hline Pinnularia mesolepta (Ehrenberg) Smith & $2,39,40$ \\
\hline Pinnularia microstauron (Ehrenberg) Cleve & 2,27 \\
\hline Pinnularia obscura Krasske & 2 \\
\hline Pinnularia rupestris Hantzsch: & 39,40 \\
\hline Pinnularia subcapitata Gregory & 40 \\
\hline Pinnularia termitina (Ehrenberg) Patrick & 27 \\
\hline \multicolumn{2}{|l|}{ Placoneis constans (Hustedt) Cox } \\
\hline$\equiv$ Navicula constans Hustedt & 25,27 \\
\hline \multicolumn{2}{|l|}{ Placoneis disparilis (Hustedt) Metzeltin \& Lange-Bertalot } \\
\hline$\equiv$ Navicula disparilis Hustedt & 27 \\
\hline Pleurosira laevis (Ehrenberg) Compère & 40 \\
\hline \multicolumn{2}{|c|}{ Pseudostaurosira elliptica (Schumann) Edlund, Morales \& Spaulding } \\
\hline$\equiv$ Staurosira elliptica (Schumann) Williams \& Round & 1 \\
\hline Sellaphora pupula (Kützing) Mereschkovsky & $2,23,25,27$ \\
\hline$\equiv$ Navicula pupula Kützing & 27 \\
\hline Stauroneis phoenicenteron (Nitzsch) Ehrenberg & $27,40^{*}$ \\
\hline Stenopterobia pelagica Hustedt & 2 \\
\hline Surirella angusta Kützing & $7,14,27$ \\
\hline Surirella cf. linearis Smith & $29,39,40$ \\
\hline Surirella robusta Ehrenberg & 14 \\
\hline Surirella tenera var. nervosa Schmidt & 39,40 \\
\hline Terpsinoё musica Ehrenberg & 24 \\
\hline Thalassiosira weissflogii (Grunow) Fryxell \& Hasle & $39,40,41$ \\
\hline \multicolumn{2}{|l|}{ Ulnaria delicatissima (Smith) Aboal \& Silva } \\
\hline$\equiv$ Fragilaria delicatissima (Smith) Lange-Bertalot & 14 \\
\hline Ulnaria ulna (Nitzsch) Compère & $1,6,7,10,11,14,22,23,24,25^{*}$ \\
\hline 三Synedra ulna (Nitzsch) Ehrenberg & $2,34,40,41$ \\
\hline Urosolenia eriensis (Smith) Round \& Crawford & $29,39,40,41$ \\
\hline Urosolenia eriensis var. morsa (West \& West) & 39,40 \\
\hline Urosolenia longiseta (Zacharias) Round \& Crawford & $39,40,41$ \\
\hline \multicolumn{2}{|l|}{ Chlorophyceae } \\
\hline Actinastrum aciculare Playfair & 40 \\
\hline Actinastrum gracillimum Smith & 39,40 \\
\hline
\end{tabular}


Table 2 (continuation)

\begin{tabular}{|c|c|}
\hline Family/Species & Reference \\
\hline Actinastrum hantzschii Lagerheim & 40,41 \\
\hline \multicolumn{2}{|l|}{ Acutodesmus acuminatus (Lagerheim) Tsarenko } \\
\hline$=$ Scenedesmus acuminatus (Lagerheim) Chodat & 40,41 \\
\hline Ankistrodesmus acerosus Komárek \& Comas González & 40 \\
\hline \multicolumn{2}{|l|}{ Ankistrodesmus arcuatus Korshikov } \\
\hline$\equiv$ Monoraphidium arcuatum (Korshikov) Hindák & 14,28 \\
\hline Ankistrodesmus bernardii Komárek & 40 \\
\hline Ankistrodesmus bibraianus (Reinsch) Korshikov & 40 \\
\hline Ankistrodesmus densus Korshikov & 40 \\
\hline Ankistrodesmus falcatus (Corda) Ralfs & $2,9,16,40^{*}$ \\
\hline Ankistrodesmus fusiformis Corda: & $2,16,23,27,39,40^{*}$ \\
\hline \multicolumn{2}{|l|}{ Ankistrodesmus gracilis (Reinsch) Korshikov } \\
\hline$=$ Selenastrum gracile Reinsch & $39,40,41$ \\
\hline Ankistrodesmus spiralis (Turner) Lemmermman & 40,41 \\
\hline Ankistrodesmus tortus Komárek \& Comas & 40 \\
\hline Ankistrodesmus turneri (West \& West) Komárek \& Comas & 40 \\
\hline Ankyra ancora (Smith) Fott & 40 \\
\hline Ankyra judayi (Smith) Fott & $1,40 *$ \\
\hline Ankyra ocellata (Korshikov) Fott & 40 \\
\hline Aphanochaete repens Braun & $2,16,27$ \\
\hline Bicuspidella sessilis Fott & 1 \\
\hline Botryococcus braunii Kützing & 40,41 \\
\hline Botryococcus protuberans West \& West & 39,40 \\
\hline Chaetophora elegans (Roth) Agardh & 7,27 \\
\hline Chaetosphaeridium cf. globosum (Nordstedt) Klebahn & 2 \\
\hline Characiellopsis skujae (Fott) Komárek & 2,27 \\
\hline Characium ambiguum Hermann & 2 \\
\hline Characium indicum Patel \& Isabella & 2 \\
\hline Characium ornithocephalum Braun & $2,9,27$ \\
\hline Chlorella vulgaris (Beyerinck) Beijerinck & 16 \\
\hline Chloromonas bichlora Ettl & 40 \\
\hline Chloromonas paradoxa Korshikov & $23,27,28$ \\
\hline Closteriopsis acicularis (Chodat) Belcher \& Swale & 40 \\
\hline Closteriopsis acicularis var. africana Hindák & 39,40 \\
\hline Closteriopsis cf. scolia Comas & $30,40,41$ \\
\hline Closteriopsis langerheimi Lemmermann & 40 \\
\hline Closteriopsis longissima (Lemmermann) Lemmermann & $2,40^{*}$ \\
\hline Coelastrum astroideum De Notaris & 40 \\
\hline Coelastrum cambricum Archer & $7,27,40^{*}$ \\
\hline Coelastrum microporum Nägeli & $9,40^{*}$ \\
\hline Coelastrum pseudomicroporum Korshikov & 40,41 \\
\hline Coelastrum pulchrum Schmidle & 40 \\
\hline
\end{tabular}


Table 2 (continuation)

\begin{tabular}{|c|c|}
\hline Family/Species & Reference \\
\hline Coelastrum reticulatum (Dangeard) Senn & 40,41 \\
\hline Coenochloris hindakii Komárek & 40,41 \\
\hline Coenochloris planconvexa Hindák & 40 \\
\hline Coenocystis asymmetrica Komárek & 18 \\
\hline Coleochaete cf. orbicularis Pringsheim & 27 \\
\hline Coleochaete irregularis Pringsheim & 1 \\
\hline Coleochaete orbicularis Pringsheim & 2 \\
\hline Crucigenia fenestrata (Schmidle) Schmidle & 40 \\
\hline Crucigenia quadrata Morren & 40 \\
\hline Crucigenia tetrapedia (Kirchner) West \& West & $16,27,28,40,41^{*}$ \\
\hline Crucigeniella apiculata (Lemmermman) Komárek & 40 \\
\hline Crucigeniella pulchra (West \& West) Komárek & 40,41 \\
\hline Desmodesmus abundans (Kirchner) Hegewald & 40 \\
\hline Desmodesmus armatus var. armatus (Chodat) Hegewald & 40 \\
\hline \multicolumn{2}{|l|}{ Desmodesmus armatus var. bicaudatus (Guglielmetti) Hegewald } \\
\hline $\equiv$ Scenedesmus bicaudatus (Hansgirg) Chodat & 40 \\
\hline Desmodesmus armatus var. spinosus (Fritsch \& Rick) Hegewald & 40 \\
\hline Desmodesmus brasiliensis (Bohlin) Hegewald & $9,12,18$ \\
\hline Desmodesmus cf. lefevrei (Deflandre) An, Friedl \& Hegewald & 40 \\
\hline \multicolumn{2}{|l|}{ Desmodesmus brevispina (Smith) Chodat } \\
\hline$=$ Scenedesmus brevispina $($ Smith) Chodat & 39 \\
\hline \multicolumn{2}{|l|}{ Desmodesmus spinosus (Chodat) Hegewald } \\
\hline$\equiv$ Scenedesmus cf. spinosus Chodat & 24 \\
\hline Desmodesmus communis (Hegewald) Hegewald & 9 \\
\hline$=$ Scenedesmus quadricauda Corda & $39,40,41$ \\
\hline Desmodesmus denticulatus (Lagerheim) An, Friedl \& Hegewald & 9 \\
\hline 三Scenedesmus denticulatus Lagerheim & 2,27 \\
\hline \multicolumn{2}{|l|}{$\begin{array}{l}\text { Desmodesmus denticulatus var. denticulatus (Lagerheim) An, } \\
\text { Friedl \& Hegewald }\end{array}$} \\
\hline 三Scenedesmus denticulatus Lagerheim & 40,41 \\
\hline Desmodesmus denticulatus var. fenestratus (Teiling) Hegewald & 40 \\
\hline Desmodesmus denticulatus var. linearis (Hansgirg) Hegewald & 40 \\
\hline Desmodesmus intermedius var. intermedius (Chodat) Hegewald & 40 \\
\hline Desmodesmus maximus (West \& West) Hegewald & 40 \\
\hline Desmodesmus protuberans (Fritsch \& Rich) Hegewald & 40 \\
\hline Desmodesmus serratus (Corda) An, Friedl \& Hegewald & 40 \\
\hline Dictyosphaerium ehrenbergianum Nageli & $2,39,40,41 *$ \\
\hline Dictyosphaerium elegans Bachmann & $39,40,41$ \\
\hline Dictyosphaerium sphagnale Hindák & 40 \\
\hline Dictyosphaerium tetrachotomum Printz & 40 \\
\hline Dimorphococcus cordatus Wolle & 40 \\
\hline Dimorphococcus lunatus Braun & $2,27,40^{*}$ \\
\hline
\end{tabular}


Table 2 (continuation)

\begin{tabular}{|c|c|}
\hline Family/Species & Reference \\
\hline \multicolumn{2}{|l|}{ Drepanochloris uherkovichii Marvan, Komárek \& Comas } \\
\hline$=$ Drepanochloris uherkovichii Marvan & 28 \\
\hline Elakatothrix gelatinosa Wille & 40 \\
\hline Elakatothrix viridis (Snow) Printz & 40 \\
\hline Eudorina elegans Ehrenberg & 40 \\
\hline Eutetramorus cf. polycoccus (Korshikov) Komárek & 40 \\
\hline Eutetramorus fottii (Hindák) Komárek & 40,41 \\
\hline Eutetramorus globosus Walton & 40 \\
\hline Eutetramorus planctonicus (Korshikov) Bourrelly & 39,40 \\
\hline Fusola cf. viridis Snow & 2 \\
\hline Fusola viridis Snow & 40 \\
\hline Gloeocystis cf. vesiculosa Nägeli & 2,25 \\
\hline Golenkinia paucispina West \& West & 40 \\
\hline Golenkinia radiata Chodat & 40,41 \\
\hline Hindakochloris insularis Comas & 40 \\
\hline Kirchneriella contorta Schmidle Bohlin & 40 \\
\hline Kirchneriella contorta var. elongata (Smith) Komárek & 40 \\
\hline Kirchneriella cornuta Korshikov & 40 \\
\hline Kirchneriella dianae (Bohlin) Comas & 40 \\
\hline Kirchneriella irregularis (Smith) Korshikov & 39,40 \\
\hline Kirchneriella lunaris (Kirchner) Möbius & $27,40,41^{*}$ \\
\hline Kirchneriella obese (West) West \& West & $2,27,39,40 *$ \\
\hline \multicolumn{2}{|l|}{$\begin{array}{l}\text { Klebsormidium subtile (Kützing) Mikhailyuk, Glaser, Holzinger } \\
\text { \& Karsten }\end{array}$} \\
\hline$\equiv$ Ulothrix subtilissima Rabenhorst & 27 \\
\hline Lagerheimia chodatii Bernard & 40 \\
\hline Lagerheimia ciliata (Lagerheim) Chodat & 40 \\
\hline \multicolumn{2}{|l|}{ Messastrum gracile (Reinsch) Garcia } \\
\hline$\equiv$ Ankistrodesmus gracilis (Reinsch) Korshikov & 2 \\
\hline$\equiv$ Selenastrum gracile Reinsch & 2,24 \\
\hline Micractinium bornhemiense (Conrad) Korshikov & 40 \\
\hline Micractinium pusillum Fresenius & 40,41 \\
\hline \multicolumn{2}{|l|}{ Monactinus simplex (Meyen) Corda } \\
\hline$\equiv$ Pediastrum simplex var. simplex Meyen & 40 \\
\hline Monoraphidium arcuatum (Korshikov) Hindák & $39,40,41$ \\
\hline Monoraphidium caribeum Hindák & 40 \\
\hline Monoraphidium cf. contortum (Thuret) Komárková-Legnerová & 28 \\
\hline Monoraphidium cf. griffithii (Berkeley) Komárková-Legnerová & 2 \\
\hline Monoraphidium circinale Nygaard & $14,40 *$ \\
\hline Monoraphidium contortum (Thuret) Komárková-Legnerová & $14,30,39,40^{*}$ \\
\hline Monoraphidium convolutum (Corda) Komárková-Legnerová & $30,39,40$ \\
\hline Monoraphidium fontinali Hindák & 39,40 \\
\hline Monoraphidium griffithii (Berkeley) Komárková-Legnerová & $14,30,40^{*}$ \\
\hline
\end{tabular}


Table 2 (continuation)

\begin{tabular}{|c|c|}
\hline Family/Species & Reference \\
\hline Monoraphidium irregulare (Smith) Komárková-Legnerová & $39,40,41$ \\
\hline Monoraphidium komarkovae Nygaard & 39,40 \\
\hline Monoraphidium minutum (Nägeli) Komárková-Legnerová & $1,39,40^{*}$ \\
\hline Monoraphidium pusillum (Printz) Komárková-Legnorová & $27,40^{*}$ \\
\hline Monoraphidium tortile (West \& West) Komárková-Legnerová & $39,40,41$ \\
\hline \multicolumn{2}{|l|}{ Mucidosphaerium pulchellum (Wood) Bock, Proschold \& Krienitz } \\
\hline$\equiv$ Dictyosphaerium pulchellum Wood & $2,9,27,39,40,41^{*}$ \\
\hline Nephrocytium limneticum (Smith) Smith & 40 \\
\hline Oocystis borgei Snow & $39,40,41$ \\
\hline Oocystis lacustris Chodat & $39,40,41$ \\
\hline Oocystis solitaria Wittrock & 40 \\
\hline$=$ Oocystis crassa Wittrock & 27 \\
\hline Pachycladella komarekii (Fott \& Kovácik) Reymond & 40 \\
\hline Pandorina morum (Muller) Bory & 40,41 \\
\hline Paradoxia multiseta Svirenko & 40,41 \\
\hline Pediastrum cf. argentinense Bourrelly \& Tell & 2 \\
\hline Pediastrum duplex var. duplex Meyen & 40,41 \\
\hline Pediastrum duplex Meyen & $14,16,23,27$ \\
\hline Pediastrum obtusum Lucks & 40 \\
\hline Phythelios viridis Frenzel & 27 \\
\hline Pithophora oedogonia (Montagne) Wittrock & 5 \\
\hline Pleudorina sphaerica Iyngar & 40 \\
\hline Pseudobohlinia americana Bourrelly & 40 \\
\hline Quadrigula closterioides (Bohlin) Printz & 39,40 \\
\hline Radiococcus planctonicus Lund & 40 \\
\hline Raphidocelis contorta (Schmidle) Marvan, Komárek \& Comas & 40 \\
\hline Rhombocystis complanata Komárek & 40 \\
\hline Scenedesmus acunae Comas & 40 \\
\hline Scenedesmus alternans Reinsch & 40 \\
\hline Scenedesmus arcuatus (Lemmermann) Lemmermann & 1 \\
\hline Scenedesmus arcuatus var. platydiscus Smith & 39,40 \\
\hline Scenedesmus bernardii Smith. & 40 \\
\hline Scenedesmus bicaudatus Dedusenko & 2,27 \\
\hline Scenedesmus bijugus (Turpin) Lagerheim & 2,7 \\
\hline Scenedesmus brevispina (Smith) Chodat & 21 \\
\hline Scenedesmus ellipticus Corda & 39,40 \\
\hline Scenedesmus heteracanthus Guerra & 40 \\
\hline Scenedesmus javanensis Chodat & 40 \\
\hline Scenedesmus linearis Komárek & 40 \\
\hline Scenedesmus microspina Chodat & 40 \\
\hline \multicolumn{2}{|l|}{ Scenedesmus obtusus Meyen } \\
\hline$=$ Scenedesmus ovalternus Chodat & $27,40^{*}$ \\
\hline
\end{tabular}


Table 2 (continuation)

\begin{tabular}{|c|c|}
\hline Family/Species & Reference \\
\hline Scenedesmus quadricauda (Turpin) Brébisson & 27 \\
\hline Scenedesmus rarciborskii Woloszynska & 7 \\
\hline Scenedesmus seelyanum Wolle & 24 \\
\hline \multicolumn{2}{|l|}{ Scenedesmus subspicatus Chodat } \\
\hline$=$ Scenedesmus cf. gutwinskii Chodat & 24 \\
\hline Schroederia antillarum Komárková & 40 \\
\hline Schroederia nitzschioides (West) Kors & 40 \\
\hline Schroederia setigera (Schröder) Lemmermann & $2,39,40 *$ \\
\hline Selenastrum bibraianum Reinsch & 40 \\
\hline \multicolumn{2}{|c|}{ Selenodyctium brasiliense (Uherkovich \& Schmidt) Comas \& Komárek } \\
\hline$=$ Kirchneriella roselata Hindák & 40 \\
\hline Sorastrum americanum (Bohlin) Schmidle & 27 \\
\hline Sorastrum spinulosum Nägeli & 40 \\
\hline Sphaerellopsis agloe Pascher & 29,40 \\
\hline Sphaerocystis schroeteri Chodat & 1 \\
\hline \multicolumn{2}{|l|}{ Stauridium tetras (Ehrenberg) Hegewald } \\
\hline$\equiv$ Pediastrum tetras (Ehrenberg) Ralfs & $2,7,14,27,40,41$ \\
\hline Stigeoclonium farctum Berthold & 27 \\
\hline Stigeoclonium tenue (Agardh) Kützing & 27 \\
\hline Tetrachlorella alternans (Smith) Korshikov & 40 \\
\hline Tetradesmus cf. obliquus (Turpin) Wynne & 24 \\
\hline \multicolumn{2}{|l|}{ Tetradesmus dimorphus (Turpin) Wynne } \\
\hline 三Scenedesmus dimorphus (Turpin) Kützing & 2 \\
\hline \multicolumn{2}{|l|}{ Tetradesmus lagerheimii Wynne \& Guiry } \\
\hline$=$ Scenedesmus acuminatus (Lagerheim) Chodat & 2,27 \\
\hline \multicolumn{2}{|l|}{ Tetradesmus obliquus (Turpin) Wynne } \\
\hline$\equiv$ Scenedesmus obliquus (Turpin) Kützing & $2,39,40^{*}$ \\
\hline Tetraedron caudatum (Corda) Hansgirg & 40 \\
\hline Tetraedron minimum (Braun) Hansgirg & $23,39,40^{*}$ \\
\hline Tetraedron triangulare Korshikov & 40 \\
\hline Tetrallantos lagerheimii Teiling & $2,40^{*}$ \\
\hline Tetrastrum elegans Playfair & 40 \\
\hline Tetrastrum heteracanthum (Nordstedt) Chodat & 40 \\
\hline Tetrastrum homoiacanthum (Hüber-Pestalozzi) Hindák & 40 \\
\hline Tetrastrum komarekii Hindák & 40 \\
\hline Tetrastrum triangulare (Chodat) Komárek & 40 \\
\hline Thorakochloris nygardii Komárek & 40,41 \\
\hline Treubaria quadrispina (Smith) Fott \& Kovácik & 40 \\
\hline Treubaria schmidlei (Schröder) Fott \& Kovácik & 40 \\
\hline Treubaria setigera (Archer) Smith & 40 \\
\hline Treubaria triappendiculata Bernard & 40 \\
\hline Ulothrix aequalis Kützing & 27 \\
\hline
\end{tabular}


Table 2 (continuation)

\begin{tabular}{|c|c|}
\hline Family/Species & Reference \\
\hline Uronema confervicola Lagerheim & 1 \\
\hline Uronema intermedium Bourrelly & 2,27 \\
\hline Volvox aureus Ehrenberg & 40 \\
\hline Westella botryoides (West) De Wild & 40 \\
\hline \multicolumn{2}{|l|}{ Willea crucifera (Wolle) John, Wynne \& Tsarenko } \\
\hline ECrucigeniella crucifera (Wolle) Komárek & $23,27,40^{*}$ \\
\hline Willea irregularis (Wille) Schmidle & 40 \\
\hline \multicolumn{2}{|l|}{ Willea rectangularis (Braun) John, Wynne \& Tsarenko } \\
\hline$\equiv$ Crucigeniella rectangularis (Nägeli) Komárek & $1,39 *$ \\
\hline \multicolumn{2}{|l|}{ Chrysophyceae } \\
\hline Bicosoeca synoica Skuja & 2 \\
\hline Chromulina cf. echinocystis Conrad & 39,40 \\
\hline Dinobryon bavaricum Imhof & 40 \\
\hline Dinobryon divergens Imhof & $29,39,40$ \\
\hline Dinobryon sertularia Ehrenberg & $27,39,40,41^{*}$ \\
\hline Salpingoeca marsonii Lemmermman & 27 \\
\hline Synura uvella Ehrenberg & 40 \\
\hline \multicolumn{2}{|l|}{ Cryptophyceae } \\
\hline Chroomonas acuta Utermöhl & $39,40,30$ \\
\hline Chroomonas nordstedtii Hansgirg & 39,40 \\
\hline Cryptomonas brasiliensis Castro, Bicudo \& Bicudo & $30,39,40,41$ \\
\hline Cryptomonas cf. tenuis Pascher & 10 \\
\hline Cryptomonas curvata Ehrenberg & 39,40 \\
\hline Cryptomonas erosa Ehrenberg & 30 \\
\hline Cryptomonas marssonii Skuja & $28,30,34,38,40^{*}$ \\
\hline Cryptomonas ovata Ehrenberg & 40 \\
\hline Cryptomonas tenuis Pascher & 9 \\
\hline Rhodomonas lacustris Pascher \& Ruttner & 39,40 \\
\hline \multicolumn{2}{|l|}{ Cyanophyceae } \\
\hline Anabaena cylindrica Lemmermann & $2,20,21$ \\
\hline$=$ Anabaena subcylindrica Borge & 21 \\
\hline Anabaena macrospora Klebahn & 40 \\
\hline Anabaena sphaerica Bornet \& Flahault & 20,21 \\
\hline Anabaena viguieri Denis \& Frémy & 40 \\
\hline \multicolumn{2}{|l|}{ Aphanizomenon flos-aquaea Ralfs ex Bornet \& Flahault } \\
\hline$\equiv$ Aphanizomenon flosaquae Ralfs ex Bornet \& Flahault & 21 \\
\hline Aphanizomenon gracile Lemmermann & 40 \\
\hline Aphanocapsa cf. grevillei (Berkeley) Rabenhorst & 23 \\
\hline Aphanocapsa delicatissima West \& West & 40 \\
\hline Aphanocapsa holsatica (Lemmermann) Cronberg \& Komárek & 26 \\
\hline Aphanocapsa incerta (Lemmermann) Cronberg \& Komárek & 18 \\
\hline \multicolumn{2}{|l|}{ Aphanocapsa koordersii Ström } \\
\hline$=$ Aphanocapsa planctonica (Smith) Komárek \& Anagnostidis & 40 \\
\hline
\end{tabular}


Table 2 (continuation)

\begin{tabular}{|c|c|}
\hline Family/Species & Reference \\
\hline Aphanocapsa parasitica (Kützing) Komárek \& Anagnostidis & $2,9,10,12,18,20,21$ \\
\hline Aphanocapsa rivularis (Carmichael) Rabenhorst & 2 \\
\hline Aphanothece clathrata West \& West & 40 \\
\hline Aphanothece microscopica Nägeli & 18,21 \\
\hline Borzia trilocularis Cohn (Gomont) & $2,18,20,21$ \\
\hline Calothrix brevissima West & $2,16,20,21,27$ \\
\hline Calothrix cylindrica Frémy & 21 \\
\hline Calothrix epiphytica West \&West & 2 \\
\hline Calothrix fusca Bornet \& Flahault & 27 \\
\hline Chamaesiphon investiens Skuja & 9,18 \\
\hline Chroococcus aphanocapsoides Skuja & 16 \\
\hline Chroococcus cf. major Komarék \& Komákova-Legnerová & 21 \\
\hline Chroococcus disperses (Keissler) Lemmermann & $1,2,18,21,36,40^{*}$ \\
\hline Chroococcus minor (Kützing) Nägeli & $9,10,18,21$ \\
\hline Chroococcus minutus (Kützing) Nägeli & $9,10,18,20,21,27,28$ \\
\hline Coelosphaerium confertum West \& West & $36,40,41$ \\
\hline Cyanostylon microcystoides Geitler & 18 \\
\hline \multicolumn{2}{|c|}{ Cylindrospermopsis raciborskii (Woloszynska) Seenayya \& Subba Raju } \\
\hline$=$ Cylindrospermopsis raciborskii (Woloszynska) & $2,20,21,36,40,41^{*}$ \\
\hline Cylindrospermum muscicola Kützing ex Bornet \& Flahault & $20,21,27$ \\
\hline \multicolumn{2}{|l|}{ Dasygloea lamyi (Gomont ex Gomont) Senna \& Komárek } \\
\hline$\equiv$ Schizothrix lamyi Gomont ex Gomont & $2,20,21,27$ \\
\hline \multicolumn{2}{|l|}{$\begin{array}{l}\text { Desmonostoc muscorum (Agardh ex Bornet \& Flahault) Hrouzek \& } \\
\text { Ventura }\end{array}$} \\
\hline$\equiv$ Nostoc muscorum Agardh ex Bornet \& Flahault & 20 \\
\hline$=$ Nostoc cf. muscorum Agardh ex Bornet \& Flahault & 2 \\
\hline \multicolumn{2}{|c|}{ Dolichospermum affine (Lemmermann) Wacklin, Hoffmann \& Komárek } \\
\hline$\equiv$ Anabaena affinis (Lemmermann) & $2,20,21$ \\
\hline \multicolumn{2}{|c|}{$\begin{array}{l}\text { Dolichospermum circinalis (Rabenhorst ex Bornet \& Flahault) Wacklin, } \\
\text { Hoffmann \& Komárek }\end{array}$} \\
\hline$\equiv$ Anabaena circinalis Rabenhorst ex Bornet \& Flahault & $30,32,34,36,40,41$ \\
\hline \multicolumn{2}{|l|}{$\begin{array}{l}\text { Dolichospermum planctonicum (Brunnthaler) Wacklin, Hoffmann \& } \\
\text { Komárek }\end{array}$} \\
\hline$\equiv$ Anabaena planctonica Brunnthaler & $29,34,30,40$ \\
\hline \multicolumn{2}{|c|}{ Dolichospermum sigmoideum (Nygaard) Wacklin, Hoffmann \& Komárek } \\
\hline$=$ Anabaena circinalis Rabenhorst $e x$ Bornet \& Flahault & 27,28 \\
\hline \multicolumn{2}{|c|}{ Dolichospermum solitarium (Klebahn) Wacklin, Hoffmann \& Komárek } \\
\hline$\equiv$ Anabaena solitaria Klebahn & $27,29,32,36,40$ \\
\hline \multicolumn{2}{|c|}{ Dolichospermum spiroides (Klebahn) Wacklin, Hoffmann \& Komárek } \\
\hline$\equiv$ Anabaena spiroides Klebahn & $29,32,36,40,41$ \\
\hline Geitleribactron periphyticum Komárek & 25 \\
\hline Geitleribactron subaequale (Geitler) Komárek & 24 \\
\hline
\end{tabular}


Table 2 (continuation)

\begin{tabular}{|c|c|}
\hline Family/Species & Reference \\
\hline Geitlerinema amphibium (Agardh ex Gomont) Anagnostidis & 24 \\
\hline $\begin{aligned}= & \text { Phormidium amphibium (Agardh ex Gomont) Anagnostidis \& } \\
& \text { Komárek }\end{aligned}$ & 21 \\
\hline = Geitlerinema unigranulatum (Singh) Komárek \& Azevedo & 21 \\
\hline$=$ Oscillatoria amphibia Agardh ex Gomont: & 20,27 \\
\hline \multicolumn{2}{|l|}{ Geitlerinema splendidum (Greville ex Gomont) Anagnostidis* } \\
\hline$=$ Geitlerinema splendidum Anagnostidis & $2,20,21$ \\
\hline$\equiv$ Oscillatoria splendida Greville ex Gomont & $20,27,41$ \\
\hline Gloeocapsa gelatinosa Kützing & 18 \\
\hline Gloeocapsa puntacta Nägeli & 40 \\
\hline Gloeocapsa siderochlamys (Skuja) Starmach & 2 \\
\hline Gloeotrichia echinulata Richter & 25 \\
\hline Hapalosiphon arboreus West \& West & $2,20,21$ \\
\hline Hapalosiphon cf. hibernicus West \& West & 2 \\
\hline Hapalosiphon luteolus West \& West & 2,20 \\
\hline Heteroleibleinia kuetzingii (Schmidle) Compère & 21 \\
\hline \multicolumn{2}{|l|}{ Heteroleibleinia pusilla (Hansgirg) Compère: 25} \\
\hline Jaaginema cf. subtilissimum (Kützing ex Forti) Anagnostidis \& Komárek & 21 \\
\hline Jaaginema cf. thermale Anagnostidis & 21 \\
\hline \multicolumn{2}{|l|}{ Jaaginema geminatum (Schwabe ex Gomont) Anagnostidis \& Komárek } \\
\hline$\equiv$ Oscillatoria geminata Schwabe ex Gomont: & 20,27 \\
\hline \multicolumn{2}{|l|}{ Jaaginema geminatum (Schwabe ex Gomont) Anagnostidis \& Komárek } \\
\hline = Jaaginema geminatum Anagnostidis \& Komárek & $20,21,23,27$ \\
\hline Jaaginema homogeneum (Frémy) Anagnostidis \& Komárek & 40 \\
\hline Jaaginema pseudogeminatum (Schmid) Anagnostidis \& Komárek & 21 \\
\hline $\begin{array}{l}\text { Jaaginema quadripunctulatum (Brühl \& Biswas) Anagnostidis \& } \\
\text { Komárek }\end{array}$ & $2,20,21$ \\
\hline \multicolumn{2}{|l|}{ Kamptonema proteus (Skuja) Strunecký, Komárek \& Smarda } \\
\hline$\equiv$ Oscillatoria proteus Skuja & 21 \\
\hline Komvophoron crassum (Vozzhennikova) Anagnostidis \& Komárek & 21 \\
\hline Komvophoron schmidlei (Jaag) Anagnostidis \& Komárek & 20,21 \\
\hline \multicolumn{2}{|l|}{ Leibleinia epiphytica (Hieronymus) Compère } \\
\hline$=$ Leibleinia epiphytica (Anagnostidis \& Komárek) & $9,10,11,20,21,23,24,25$ \\
\hline$\equiv$ Lyngbya epiphytica Hieronymus & 27 \\
\hline Leibleinia subtilis (Holden) Anagnostidis \& Komárek & 21 \\
\hline \multicolumn{2}{|l|}{ Lemmermanniella obesa Azevedo, Souza \& Menezes } \\
\hline$=$ Leibleinia pellucida $($ Umezaki) & 2 \\
\hline Leptolyngbya angustissima (West \&West) Anagnostidis \& Komárek & 10,24 \\
\hline Leptolyngbya foveolara (Gomont) Anagnostidis \& Komárek & $9,10,24,25$ \\
\hline Leptolyngbya foveolarum (Gomont) Hansgirg & $9,10,24,25$ \\
\hline Leptolyngbya fragilis (Gomont) Anagnostidis \& Komárek & 9,21 \\
\hline$\equiv$ Lyngbya fragilis (Gomont) Compère & 25 \\
\hline
\end{tabular}


Table 2 (continuation)

\begin{tabular}{|c|c|}
\hline Family/Species & Reference \\
\hline $\begin{array}{l}\text { Leptolyngbya lagerheimii (Gomont ex Gomont) Anagnostidis \& } \\
\text { Komárek }\end{array}$ & 25 \\
\hline$\equiv$ Lyngbya lagerheimii Gomont & 21,27 \\
\hline \multicolumn{2}{|l|}{ Leptolyngbya lurida (Gomont) Anagnostidis \& Komárek } \\
\hline$\equiv$ Phormidium luridum Gomont & 25 \\
\hline$=$ Lyngbya gomontiana Senna & 21 \\
\hline \multicolumn{2}{|l|}{ Leptolyngbya perelegans (Lemmermann) Anagnostidis \& Komárek } \\
\hline$=$ Leptolyngbya perelegans (Lemmermann) & $1,2,9,10,11,20,21,23,24,25$ \\
\hline \multicolumn{2}{|l|}{ Leptolyngbya polysiphoniae (Frémy) Anagnostidis } \\
\hline$=$ Lyngbya $\mathrm{cf}$. polysiphonae Frémy & 20 \\
\hline Leptolyngbya purpurascens (Gomont) Anagnostidis \& Komárek & 21 \\
\hline Leptolyngbya ramosa (Petersen) Anagnostidis \& Komárek & 21 \\
\hline Leptolyngbya subtilis (West) Anagnostidis & 25 \\
\hline Leptolyngbya tenuis (Gomont) Anagnostidis \& Komárek & 2,25 \\
\hline$=$ Lyngbya comperei Senna & 21 \\
\hline \multicolumn{2}{|l|}{ Leptolyngbya thermalis Anagnostidis } \\
\hline$=$ Leptolyngbya thermalis $($ Lemmermann $)$ & $2,20,21,40^{*}$ \\
\hline Leptolyngbya valderiana (Gomont) Anagnostidis \& Komárek & 21 \\
\hline \multicolumn{2}{|l|}{$\begin{array}{l}\text { Limnococcus limneticus (Lemmermann) Komárková, Jezberová, } \\
\text { Komárek \& Zapomelová) }\end{array}$} \\
\hline$\equiv$ Chroococcus limneticus Lemmermann & $9,18,21,36,40^{*}$ \\
\hline Limnothrix mirabilis (Böcher) Anagnostidis & 25 \\
\hline Lyngbya cf. diguetii Gomont & 2 \\
\hline Lyngbya limnetica Lemmermann & 39 \\
\hline Lyngbya martensiana Meneghini ex Gomont & $27,36^{*}$ \\
\hline $\begin{array}{l}\equiv \text { Porphyrosiphon martensianus (Meneghini ex Gomont) Anagnostidis } \\
\text { \& Komárek }\end{array}$ & $9,10,21,24,25^{*}$ \\
\hline \multicolumn{2}{|l|}{ Lyngbya natans Hansgirg } \\
\hline$=$ Phormidium natans (Gomont) Senna \& Compère & 21 \\
\hline \multicolumn{2}{|l|}{ Lyngbya nigra Agardh ex Gomont } \\
\hline$=$ Lyngbya nigra $($ Agardh $)$ & 2,20 \\
\hline Lyngbya nordgaardii Wille & 27 \\
\hline Lyngbya pellucida Umezaki & 27 \\
\hline Merismopedia cf. minima Beck & 21 \\
\hline Merismopedia convoluta Brébisson & 36,40 \\
\hline Merismopedia duplex Playfair & $2,21,27$ \\
\hline Merismopedia glauca (Ehrenberg) Kützing & $2,21,27,36,40,41^{*}$ \\
\hline Merismopedia punctata Meyen & $2,20,23,27,36,40^{*}$ \\
\hline Merismopedia tenuissima Lemmermann & $14,21,27,36,40^{*}$ \\
\hline Microchaete tenera Thuret ex Bornet \& Flahault & 21 \\
\hline \multicolumn{2}{|l|}{ Microcoleus autumnalis (Gomont) Strunecky, Komárek \& Johansen } \\
\hline$\equiv$ Phormidium autumnale Gomont & 21 \\
\hline
\end{tabular}


Table 2 (continuation)

\begin{tabular}{l}
\hline Family/Species \\
\hline Microcoleus brasiliensis Borge \\
Microcystis aeruginosa Kützing \\
Microcystis cf. panniformis Komárek, Komárková-Legnerová, \\
Sant'Anna, Azevedo \& Senna
\end{tabular}

Microcystis smithii Komárek \& Anagnostidis

= Aphanocapsa pulchra (Kützing) Rabenhorst

Nostoc cf. commune Vaucher

Nostoc cf. spongiaeforme Agardh ex Bornet \& Flahault

Oscillatoria annae Goor

Oscillatoria cf. perornata Skuja

Oscillatoria limosa Agardh ex Gomont

Oscillatoria princeps Vaucher ex Gomont

= Oscillatoria princeps (Vaucher)

Oscillatoria rupicola (Hansgirg) Hansgirg ex Forti

Oscillatoria sancta Kützing ex Gomont

Oscillatoria simplicissima Gomont

$\equiv$ Phormidium simplicissimum (Gomont) Anagnostidis \& Komárek

= Phormidium cf. simplicissimum (Gomont) Anagnostidis \& Komárek

Oscillatoria subbrevis Schmidle

Oscillatoria subtilissima (Kützing) Gomont

Oscillatoria trichoides Szafer

Phormidesmis molle (Gomont) Turicchia, Ventura, Komárková \&

Komárek)

$\equiv$ Phormidium molle Gomont

Phormidium cf. favosum Gomont

Phormidium cf. willei (Gardner)

Phormidium foreaui (Frémy) Umezaki \& Watanabe

$\equiv$ Oscillatoria foreaui Frémy

Phormidium formosum (Bory ex Gomont) Anagnostidis \& Komárek

Phormidium granulatum (Gardner) Anagnostidis

$=$ Phormidium granulatum (Gardner)

$\equiv$ Tychonema granulatum (Gardner) Anagnostidis \& Komárek

Phormidium irriguum (Kützing ex Gomont) Anagnostidis \& Komárek

Phormidium jadinianum Gomont

Phormidium prolifica Gomont ex Gomon

Phormidium retzii Kützing ex Gomont

Phormidium tenue Gomont

Phormidium willei (Gardner) Anagnostidis \& Komárek

Planktolyngbya tallingii Komárek \& Kling

Planktothrix agardhii (Gomont) Anagnostidis \& Komárek

Planktothrix mucicola Hübber-Pestalozzi \& Naum

Planktothrix prolifica (Gomont) Anagnostidis \& Komárek

= Planktothrix prolifica (Gomont)

\section{Reference}

20, 27

$14,21,27,29,32,40,41 *$

25

40

2,27

20,27

40

$2,20,21,25,27$

25

$20,21,27$

$20,21,25$

21

$2,9,21,23,24,25,27,40^{*}$

21

21

$2,20,21,25,27$

27

21

$9,10,21,24,25$

21

20

23

27

40

20,25

21

21

21

21

21

40

$21,40 *$

21

21,40 *

40

21 
Table 2 (continuation)

Family/Species

Porphyrosiphon cf. versicolor (Gomont) Anagnostidis \& Komárek

Porphyrosiphon martensianus (Meneghini ex Gomont) Anagnostidis \& Komárek

Pseudanabaena catenata Lauterborn

Pseudanabaena cf. moniliformis Komárek \& Kling

Pseudanabaena galeata Böcher

Pseudanabaena limnetica (Lemmermann) Komárek

Pseudanabaena minima (An) Anagnostidis

Pseudanabaena moniliformis Komárek \& Kling

Pseudanabaena mucicola (Naumann \& Huber-Pestalozzi) Schwabe

Pseudanabaena skujae Claus

Radiocystis fernandoi Komárek \& Komárková-Legnerová

Raphidiopsis mediterranea Skuja

Romeria gracilis (Koczwara) Koczwara

Scytonema chiastum Geitler

Scytonema mirabile Bornet

Spirulina laxa Smith

Spirulina princeps West \&West

Stenomitos frigidus (Fritsch) Miscoe \& Johansen

$\equiv$ Pseudanabaena frigida (Fritsch) Anagnostidis

Synechococcus cf. sulphuricus Dor

Synechococcus elongatus (Nägeli) Nägeli

Synechococcus mundulus Skuja

Synechocystis aquatilis Sauvageau

Synechocystis pevalekii Ercegovic

Synechocystis salina Wislouch

Tapinothrix stagnalis (Hansgirg) Bohunická \& Johansen

$\equiv$ Homoeothrix stagnalis (Hansgirg) Komárek \& Kovácik

Trichodesmium lacustre Klebahn

Euglenophyceae

Colacium vesiculosum Ehrenberg

Cryptoglena skujae Marin \& Melkonian

= Phacus agilis Skuja

Euglena acus var. longissima Deflandre

Euglena cf. viridis (Müller) Ehrenberg

Euglena ehrenbergii Klebs

Euglena oxyuris Schmarda

Euglena spirogyra var. fusca Klebs

Euglena spirogyra var. spirogyra (Brons) Ehrenberg

Euglena tripteris (Dujardin) Klebs

Euglena viridis (Müller) Ehrenberg

= Euglena oblonga Schmitz
Reference

24

40

$2,7,14,21,23$

28

21

14

25

$2,9,21,24$

$25,40^{*}$

14,15

$14,32,34,38^{*}$

40

$14,40 *$

21

21

21

$2,21,27$

$1,9,10,24$

21

1,21

20, 21

$20,21,25,27,38,30,40^{*}$

21

40

25

20, 21

13

25

40

2

34,40

34,40

34,40

34,40

34,40

40 
Table 2 (continuation)

\begin{tabular}{|c|c|}
\hline Family/Species & Reference \\
\hline \multicolumn{2}{|l|}{ Lepocinclis acus (Müller) Marin \& Melkonian } \\
\hline$\equiv$ Euglena acus Ehrenberg & $2,23,27,34,40$ \\
\hline Lepocinclis caudata (Cunha) Pascher & 34,40 \\
\hline Lepocinclis fusiformis (Carter) Lemmermann & 34,40 \\
\hline \multicolumn{2}{|l|}{ Lepocinclis spirogyroides Marin \& Melkonian } \\
\hline$=$ Euglena spirogyra Ehrenberg & 27 \\
\hline Lepocinclis ovum (Ehrenberg) Lemmermann & 2 \\
\hline Lepocinclis ovum var. globula (Perty) Lemmermann & 34,40 \\
\hline Lepocinclis ovum var. ovum (Ehrennberg) Lemmermann & 40 \\
\hline Lepocinclis salina Fritsch & $2,34,40^{*}$ \\
\hline Lepocinclis texta var. richiana (Conrad) Hübber-Pestalozzi & 34,40 \\
\hline Lepocinclis texta var. texta (Dujardin) Lemmermann & 40 \\
\hline \multicolumn{2}{|l|}{ Monomorphina pyrum (Ehrenberg) Mereschkowsky } \\
\hline$\equiv$ Phacus pyrum (Ehrenberg) Archer & 40 \\
\hline Phacus acuminatus Stokes & $2,40^{*}$ \\
\hline Phacus acutus Pochmann & 40 \\
\hline Phacus arnoldii Swirenko & 40 \\
\hline Phacus caudatus Hübner & 40 \\
\hline Phacus cf. gigas Cunha & 40 \\
\hline Phacus cf. megalopsis Pochmann & 40 \\
\hline Phacus helicoides Pochmann & 40 \\
\hline Phacus horridus Pochmann & 34,40 \\
\hline Phacus indicus Skvortzov & 2 \\
\hline Phacus lefevrei Bourrelly & 34,40 \\
\hline Phacus longicauda var. attenuata (Pochmann) Hübber-Pestalozzi & 34,40 \\
\hline Phacus longicauda var. longicauda (Ehrenberg) Dujardin & 40 \\
\hline Phacus margaritatus Pochmann & 40 \\
\hline Phacus orbicularis Hübner & $2,34,40^{*}$ \\
\hline Phacus platalea Drezepolski & 40 \\
\hline Phacus pleuronectes (Müller) Nitzsch ex Dujardin & $27,34,40^{*}$ \\
\hline Phacus polytrophos Pochmann & 28 \\
\hline Phacus stokesii Lemmermann & 28 \\
\hline Phacus suecicus Lemmermann & 40,41 \\
\hline Phacus textus Pochmann & 40 \\
\hline Phacus tortus (Lemmermann) Skvortzov & 34,40 \\
\hline \multicolumn{2}{|l|}{ Strombomonas argentinensis Emiliani: 34, 40} \\
\hline \multicolumn{2}{|l|}{ Strombomonas ensifera var. ensifera (Daday) Deflandre: 40, 41} \\
\hline \multicolumn{2}{|l|}{ Strombomonas ensifera var. javanica Hübber-Pestalozzi: 34, 40} \\
\hline Strombomonas fluviatilis (Lemmermann) Deflandre & $7,34,40,41^{*}$ \\
\hline Strombomonas gibberosa (Playfair) Deflandre & $27,34,40^{*}$ \\
\hline Strombomonas girardiana var. girardiana (Playfair) Deflandre & 30,40 \\
\hline Strombomonas girardiana var. triondulata Tell \& Domitrovic & 34,40 \\
\hline
\end{tabular}


Table 2 (continuation)

\begin{tabular}{|c|c|}
\hline Family/Species & Reference \\
\hline Strombomonas ovalis (Playfair) Deflandre & 30,40 \\
\hline Strombomonas rotunda (Playfair) Deflandre & 30,40 \\
\hline Strombomonas verrucosa (Daday) Deflandre & 40 \\
\hline Trachelomonas abrupta Swirenko & 40 \\
\hline Trachelomonas acanthophora Stokes & 40 \\
\hline Trachelomonas allorgei Playfair & 40 \\
\hline Trachelomonas amphoriformis var. granulosa Couté \& Iltis & 40 \\
\hline Trachelomonas armata var. armata (Ehrenberg) Stein & $2,27,40^{*}$ \\
\hline Trachelomonas armata var. heterospina Swirenko & 34,40 \\
\hline Trachelomonas armata var. steinii Lemmermann & $33,34,40$ \\
\hline Trachelomonas caudata (Ehrenberg) Stein & 40 \\
\hline Trachelomonas cervicula Stokes & $33,34,40$ \\
\hline Trachemolomas cf. mucosa Swirenko & 40 \\
\hline Trachelomonas cf. varians Skvortzov & 28 \\
\hline Trachelomonas clamydophora Nygaard & 40 \\
\hline Trachelomonas conica Playfair & $25,40^{*}$ \\
\hline Trachelomonas cupula Deflandre & 34 \\
\hline Trachelomonas curta Cunha & 39 \\
\hline Trachelomonas curta var. curta Cunha & 28 \\
\hline Trachelomonas curta var. minima Tell \& Domitrovic & $33,34,40$ \\
\hline Trachelomonas cylindrica Playfair & $33,34,40$ \\
\hline Trachelomonas dangeardiana Deflandre & 40 \\
\hline Trachelomonas dastuguei Balech & $33,34,40$ \\
\hline Trachelomonas fragaria Deflandre & 40 \\
\hline Trachelomonas granulata Swirenko & 40 \\
\hline Trachelomonas hemisphaerica Emiliani & 40 \\
\hline Trachelomonas hexangulata (Swirenko) Playfair & 40 \\
\hline Trachelomonas hirta Cunha & 40 \\
\hline Trachelomonas hispida var. crenulatocollis (Maskell) Lemmermann & 40 \\
\hline Trachelomonas hispida (Perty) Stein var. duplex Deflandre & 28,40 \\
\hline Trachelomonas hispida var. hispida (Perty) Stein & $9,24,25,28,30,39 *$ \\
\hline Trachelomonas irregularis var. irregulares Swirenko & $1,40^{*}$ \\
\hline Trachelomonas lacustris var. lacustris Drezepolski & 40 \\
\hline Trachelomonas lacustris var. ovalis Drezepolski & $33,34,40$ \\
\hline Trachelomonas lemmermannii Woloszynska & 34 \\
\hline Trachelomonas levefrei Deflandre & 40 \\
\hline Trachelomonas magdaleniana Deflandre & 40 \\
\hline Trachelomonas malum Conrad & 34,40 \\
\hline Trachelomonas mangini Deflandre & 40 \\
\hline Trachelomonas megalacantha Cunha & 40 \\
\hline Trachelomonas minuscula Drezepolski & 40 \\
\hline Trachelomonas naviculiformis Deflandre & 40 \\
\hline Trachelomonas oblonga Lemmermann & $33,39,40$ \\
\hline
\end{tabular}


Table 2 (continuation)

\begin{tabular}{|c|c|}
\hline Family/Species & Reference \\
\hline Trachelomonas obovata Stokes & 40 \\
\hline Trachelomonas parvicollis Deflandre & $33,34,40$ \\
\hline Trachelomonas planctonica Swirenko & 40 \\
\hline Trachelomonas pseudobulla Swirenko & $28,40^{*}$ \\
\hline Trachelomonas pulcherrima Playfair & 40 \\
\hline Trachelomonas pusilla Playfair & $33,34,40$ \\
\hline Trachelomonas raciborskii Woloszynska & $2,40^{*}$ \\
\hline Trachelomonas robusta Swirenko & $25,40^{*}$ \\
\hline Trachelomonas rotunda Swirenko & $27,28,33,34,40^{*}$ \\
\hline Trachelomonas rugulosa Stein & $33,34,39,40$ \\
\hline Trachelomonas rugulosa Stein f. paralela Tell \& Domitrovic & $2,40^{*}$ \\
\hline Trachelomonas saccata Lemmermann & 28 \\
\hline Trachelomonas scabra Playfair & 40 \\
\hline Trachelomonas sculpta Balech & $2,33,34,40^{*}$ \\
\hline Trachelomonas similis var. similis Stokes & 40,41 \\
\hline Trachelomonas similis Stokes var. spinosa Hubber-Pestalozzi & 33,34 \\
\hline Trachelomonas superba Swirenko & 40 \\
\hline Trachelomonas varians Deflandre & 34,40 \\
\hline Trachelomonas verrucosa Stokes & $2,25,28,33,34,40^{*}$ \\
\hline Trachelomonas volvocina (Ehrenberg) Ehrenberg & $1,40 *$ \\
\hline Trachelomonas volvocinopsis Swirenko & $25,28,30,33,34,39,40,41^{*}$ \\
\hline Trachelomonas woycickii Koczwara & $33,34,40$ \\
\hline Trachelomonas woycickii var. pusilla Drezepolski & 40 \\
\hline \multicolumn{2}{|l|}{ Oedogoniophyceae } \\
\hline Oedogonium reinschii Roy ex Hirn & $2,14,23,27$ \\
\hline Oedogonium warmingianum Wittrock ex Hirn & 23,27 \\
\hline \multicolumn{2}{|l|}{ Rodophyceae } \\
\hline Audouinella pygmaea (Kützing) Weber-van Bosse & 7,27 \\
\hline \multicolumn{2}{|l|}{ Compsopogon caeruleus (Balbis ex Agardh) Montagne } \\
\hline$=$ Compsopogon leptoclados Montagne & 27 \\
\hline \multicolumn{2}{|l|}{ Xanthophyceae } \\
\hline Acanthochloris scherffelii Pascher & 8 \\
\hline Brachiogonium ophiaster Pascher \& Ettl & 31,40 \\
\hline Centritractus belenophorus Lemmermann & 31,40 \\
\hline Centritractus belonophorus (Schmidle) Lemmermann & 8 \\
\hline Characiopsis acuta (Braun) Borzì & 8,9 \\
\hline Characiopsis aquilonaris Skuja & $1,2,8,9,10,18,27$ \\
\hline Characiopsis elegans Ettl & $8,9,10$ \\
\hline Characiopsis falx Pascher & 9 \\
\hline $\begin{array}{l}\text { Characiopsis longipes (Rabenhorst) Borzi } \\
\equiv \text { Characiopsis longipes (Braun) Borzi }\end{array}$ & 2,27 \\
\hline Characiopsis microcysticola Skuja & 8 \\
\hline Characiopsis minor Pascher & 2,27 \\
\hline
\end{tabular}


Table 2 (continuation)

\begin{tabular}{|c|c|}
\hline Family/Species & Reference \\
\hline Characiopsis minutissima Pascher & 8 \\
\hline Characiopsis pyriformis (Braun) Borzi & $8,9,24,27$ \\
\hline Characiopsis sphagnicola Pascher & 9,18 \\
\hline Characiopsis subulata (Braun) Borzì & 27 \\
\hline \multicolumn{2}{|l|}{$\begin{array}{l}\text { Characiopsis subulata (Braun) Borzì var. ensiformis } \\
\text { (Hermann) Lemmermann }\end{array}$} \\
\hline$\equiv$ Characium ensiforme Hermann & 2,9 \\
\hline Gloeobotrys lunatus Ettl & 31 \\
\hline Goniochloris cochleata Pascher \& Ettl & 31,40 \\
\hline Goniochloris contorta (Bourrelly) Ettl & 31,40 \\
\hline Goniochloris fallax Fott & 40 \\
\hline Goniochloris spinosa Pascher & 31 \\
\hline Isthmochloron gracile (Reinsch) Skuja & 31,40 \\
\hline Isthmochloron lobulatum (Nägeli) Skuja & 31,40 \\
\hline Isthmochloron neustonica Zolacar \& Pizzaro & 31 \\
\hline Ophiocytium capitatum Wolle & $2,27,28,40^{*}$ \\
\hline Pseudostaurastrum enorme (Ralfs) Chodat & 31,40 \\
\hline Pseudostaurastrum limneticum (Borge) Cout \& Rous & 31 \\
\hline Tetraedriella cf. jovetii (Bourrelly) Bourrelly & 11 \\
\hline Tetraedriella jovetti (Bourrelly) Bourrelly & 31,40 \\
\hline Tetraedriella regularis (Kützing) Fott & 31,40 \\
\hline Tetraedriella spinigera Skuja & 31,40 \\
\hline Tetraplektron cf. acuminatum (Pascher) Fott & 40 \\
\hline Tetraplektron acutum (Pascher) Fott & 31,40 \\
\hline Tetraplektron laevis (Bourrelly) Ettl & 31,40 \\
\hline Tetraplektron torsum (Turner) Dedusenko-Shchegoleva & $28,31,40^{*}$ \\
\hline Tetraplektron tribulus (Pascher) Loeb & 31,40 \\
\hline \multicolumn{2}{|l|}{ Zygnemaphyceae } \\
\hline Actinotaenium cf. wollei (West \&West) Teiling & $2,40^{*}$ \\
\hline Actinotaenium cucurbitinum (Bisset) Teiling & 23,27 \\
\hline Actinotaenium globosum (Bulnheim) Förster ex Compère & 2,27 \\
\hline Bambusina brebissoni Kützing & 40 \\
\hline Closterium aciculare West & 40 \\
\hline Closterium acutum var. variable Brébisson & 39,40 \\
\hline Closterium cf. leibleinii Kützing & 40 \\
\hline Closterium cf. navicula (Brébisson) Lütkemüller & 2 \\
\hline Closterium closterioides (Ralfs) Louis \& Peeters & 2 \\
\hline Closterium dianae Ehrenberg ex Ralfs & 35 \\
\hline Closterium gracile Brébisson & $2,16,40^{*}$ \\
\hline Closterium incurvum Brébisson & $2,9,35^{*}$ \\
\hline Closterium jenneri Ralfs & $2,23,27,35,39,40^{*}$ \\
\hline Closterium kuetzingii Brébisson & $2,16,27$ \\
\hline Closterium leibleinii Kützing ex Ralfs & 7,27 \\
\hline
\end{tabular}


Table 2 (continuation)

\begin{tabular}{|c|c|}
\hline Family/Species & Reference \\
\hline Closterium limneticum Lemmermann & 40 \\
\hline Closterium lineatum Ehrenberg ex Ralfs & 35,40 \\
\hline $\begin{array}{l}\text { Closterium lunula Ehrenberg \& Hemprich ex Ralfs var. intermedium } \\
\text { Gutwinski }\end{array}$ & 18 \\
\hline Closterium malmei Borge & 2 \\
\hline Closterium moniliferum Ehrenberg ex Ralfs & 7 \\
\hline Closterium navicula (Brébisson) Lütkemüller & $9,23,27$ \\
\hline Closterium nordstedtii Chodat & 39,40 \\
\hline Closterium parvulum Nägeli & $27,40^{*}$ \\
\hline Closterium setaceum Ehrenberg ex Ralfs & $2,16,39,40^{*}$ \\
\hline Closterium subulatum (Kützing) Brébisson & 27 \\
\hline Closterium tortum Griffiths & 1 \\
\hline Closterium toxon West & 2 \\
\hline Closterium tumidum Johnson & 18 \\
\hline Closterium venus Kützing ex Ralfs & 27 \\
\hline Cosmarium abbreviatum Raciborski & $2,9,18,22,27,39,40^{*}$ \\
\hline Cosmarium angulare Johnson & 18 \\
\hline Cosmarium anisochondrum Nordstedt & 2 \\
\hline Cosmarium baileyi Wolle & 9 \\
\hline Cosmarium bioculatum var. canadense Krieger \& Gerloff & 35 \\
\hline Cosmarium biretum Brébisson ex Ralfs: & 9,27 \\
\hline Cosmarium cf granatum Brébisson ex Ralfs & 40 \\
\hline Cosmarium cf. blyttii Wille & 2 \\
\hline Cosmarium cf. hammeri Reinsch & 2 \\
\hline Cosmarium cf. ocellatum Eichler \& Gütwinski & 40 \\
\hline Cosmarium cf. ordinatum (Børgesen) West \& West & 2 \\
\hline Cosmarium cf. vexatum West & 7 \\
\hline Cosmarium commissurale Brébisson ex Ralfs & 2,7 \\
\hline Cosmarium connatum Brébisson ex Ralfs & 16 \\
\hline Cosmarium contractum Kirchner & 40 \\
\hline Cosmarium contractum var. minutum (Delponte) Coesel & 35 \\
\hline Cosmarium decoratum West \& West & 40 \\
\hline Cosmarium denticulatum Borge & $27,35,40^{*}$ \\
\hline Cosmarium galeritum Nordstedt & 18 \\
\hline Cosmarium granatum Brébisson ex Ralfs & $2,7,16,23,27$ \\
\hline Cosmarium humile Nordstedt ex Toni & 2 \\
\hline Cosmarium impressulum Elfving & $18,23,27,35^{*}$ \\
\hline Cosmarium laeve Rabenhorst & $2,9,24$ \\
\hline Cosmarium lagoense Nordstedt. var. amoebum Forst & 40 \\
\hline Cosmarium majae Strøm & 27 \\
\hline Cosmarium margaritatum (Lundell) Roy \& Biss & $1,23,27,40^{*}$ \\
\hline Cosmarium minimum West \& West & 18 \\
\hline Cosmarium naegelianum Brébisson & $2,27,43^{*}$ \\
\hline
\end{tabular}


Table 2 (continuation)

\begin{tabular}{|c|c|}
\hline Family/Species & Reference \\
\hline Cosmarium norimbergense Reinsch & 2 \\
\hline Cosmarium ornatum var. ornatum Ralfs ex Ralfs & 40 \\
\hline Cosmarium phaseolus Brébisson ex Ralfs & 23 \\
\hline Cosmarium porrectum Nordstedt & 9 \\
\hline Cosmarium porteanum Archer & 1 \\
\hline Cosmarium protractum (Nägeli) Bary & 35 \\
\hline Cosmarium pseudoconnatum Nordstedt & $2,7,16,40^{*}$ \\
\hline Cosmarium pseudopyramidatum Lundell & $2,9,18,23,27$ \\
\hline Cosmarium punctulatum Brébisson & 2,9 \\
\hline Cosmarium pyramidatum Brébisson ex Ralfs & 16 \\
\hline Cosmarium quadratulum (Gay) Toni & 16 \\
\hline Cosmarium quadrum Lundell & 27 \\
\hline Cosmarium regnellii Wille & $2,9,16,24$ \\
\hline Cosmarium regnesi Reinsch & 1,28 \\
\hline Cosmarium reniforme (Ralfs) Archer & 9,24 \\
\hline Cosmarium subadoxum Grönblad & 9,10 \\
\hline Cosmarium subspeciosum Nordstedt & 9 \\
\hline Cosmarium subtumidum var. subtumidum Wittrock & 35 \\
\hline Cosmarium triangulare Borge & 27 \\
\hline Cosmarium trilobulatum Reinsch & $1,2,9$ \\
\hline Cosmarium undulatum Corda ex Ralfs & 2 \\
\hline Cosmarium vexatum West & 9,23 \\
\hline Cylindrocystis brebissonii (Ralfs) Bary & 23,27 \\
\hline Desmidium aptogonum Brébisson ex Kützing & 27 \\
\hline Desmidium baileyi (Ralfs) Nordstedt & 27 \\
\hline Desmidium cf. coarctatum Nordestedt & 40 \\
\hline Desmidium gracilliceps (Nordstedt) Lagerh & 40 \\
\hline Desmidium laticeps Nordstedt & 40 \\
\hline Desmidium pseudostreptonema West \& West & 2 \\
\hline Desmidium swartzii (Agardh) Agardh ex Ralfs & 40 \\
\hline Euastrum abruptum Nordstedt & 35,40 \\
\hline$=$ Euastrum cf. abruptum Nordstedt & 9 \\
\hline Euastrum abruptum var. abruptum Nordstedt & 2 \\
\hline Euastrum ansatum Ehrenberg ex Ralfs & 2 \\
\hline$=$ Euastrum inusitatum Förster \& Eckert & 35,40 \\
\hline Euastrum denticulatum Gay & $2,16,23,40^{*}$ \\
\hline Euastrum elegans (Brébisson) Kützing & $27,40^{*}$ \\
\hline Euastrum gayanum Toni & 2 \\
\hline Euastrum gayanum var. angulatum Krieger & 35,40 \\
\hline $\begin{array}{l}\text { Euastrum gemmatum (Brébisson) ex Ralfs } \\
=\text { Euastrum gemmatum Ralfs }\end{array}$ & 2,27 \\
\hline Euastrum insulare (Wittrock) Roy & 40 \\
\hline Euastrum intermedium var. longicolle Borge & 35 \\
\hline
\end{tabular}


Table 2 (continuation)

\begin{tabular}{|c|c|}
\hline Family/Species & Reference \\
\hline Euastrum mononcylum (Nordstedt) Raciborski & 27 \\
\hline Euastrum porrectum Borge & 27 \\
\hline Euastrum pulchellum Brébisson & 23,27 \\
\hline Euastrum rectangulare Fritsch \& Rich & $7,40^{*}$ \\
\hline Euastrum sinuosum Lenorm ex Archer & 40 \\
\hline Gonatozygon aculeatum Hastings & 40 \\
\hline Gonatozygon kinahanii (Archer) Rabenhorst & 40 \\
\hline Gonatozygon monotaenium Bary & 2,14 \\
\hline Gonatozygon pilosum Wolle & $27,40^{*}$ \\
\hline Hyalotheca dissiliens Brébisson ex Ralfs & 40 \\
\hline$=$ Hyalotheca dissiliens (Smith) Brébisson ex Ralfs & 2,27 \\
\hline Hyalotheca mucosa (Mert) Ehrenberg ex Ralfs & 40 \\
\hline Micrasterias abrupta West \& West & $2,35^{*}$ \\
\hline Micrasterias arcuata Bailey & 27 \\
\hline Micrasterias borgei Krieg & 35 \\
\hline Micrasterias cf. abrupta West \& West & 24 \\
\hline Micrasterias cf. borgei Willi & 2 \\
\hline Micrasterias foliacea Bailey ex Ralfs & 14 \\
\hline Micrasterias furcata Agardh ex Ralfs & $27,35,40^{*}$ \\
\hline$=$ Micrasterias radiata Hassall $e x$ West $\&$ West & 2 \\
\hline Micrasterias laticeps Nordstedt & $2,27,35,40^{*}$ \\
\hline Micrasterias mahabuleshwarensis Hobs & $24,35^{*}$ \\
\hline Micrasterias radiosa Ralfs & 40 \\
\hline $\begin{array}{l}\text { Micrasterias rotata (Greve) Ralfs ex Ralfs } \\
=\text { Micrasterias rotata } \text { Ralfs }\end{array}$ & 24 \\
\hline $\begin{array}{l}\text { Micrasterias truncata (Corda) Brébisson ex Ralfs } \\
=\text { Micrasterias truncata Brébisson ex Ralfs }\end{array}$ & 24,27 \\
\hline Mougeotia recurva (Hassall) Toni & 23,27 \\
\hline $\begin{array}{l}\text { Netrium digitus (Ehrenberg) Itzigsohn \& Rothe } \\
=\text { Netrium digitus (Brébisson ex Ralfs) Itzigsohn \& Rothe }\end{array}$ & $2,23,27$ \\
\hline Octacanthium mucronulatum (Nordstedt) Compère & 24 \\
\hline$\equiv$ Arthrodesmus mucronulatus Nordstedt & 27 \\
\hline Onychonema laeve Nordstedt & 40 \\
\hline Pachyphorium taxichondrum (Lundell) Palamar-Mordvintseva & 2 \\
\hline \multicolumn{2}{|l|}{$\equiv$ Cosmarium cf. taxichondrum Lundell } \\
\hline Pleurotaenium cylindricum var. stuhlmannii Turner & 35 \\
\hline Pleurotaenium ehrenbergii (Ralfs) Bary & 2,27 \\
\hline Pleurotaenium nodosum (Bailey ex Ralfs) Lundell & 27 \\
\hline Pleurotaenium trabecula (Ehrenberg) & 35 \\
\hline Sphaerozosma laeve (Nordstedt) Thom & 40 \\
\hline$\equiv$ Onychonema laeve Nordestedt & 2,27 \\
\hline Spondylosium panduriforme (Heimerl) Teiling & 27 \\
\hline Spondylosium planum (Wolle) West \& West & 40 \\
\hline
\end{tabular}


Table 2 (continuation)

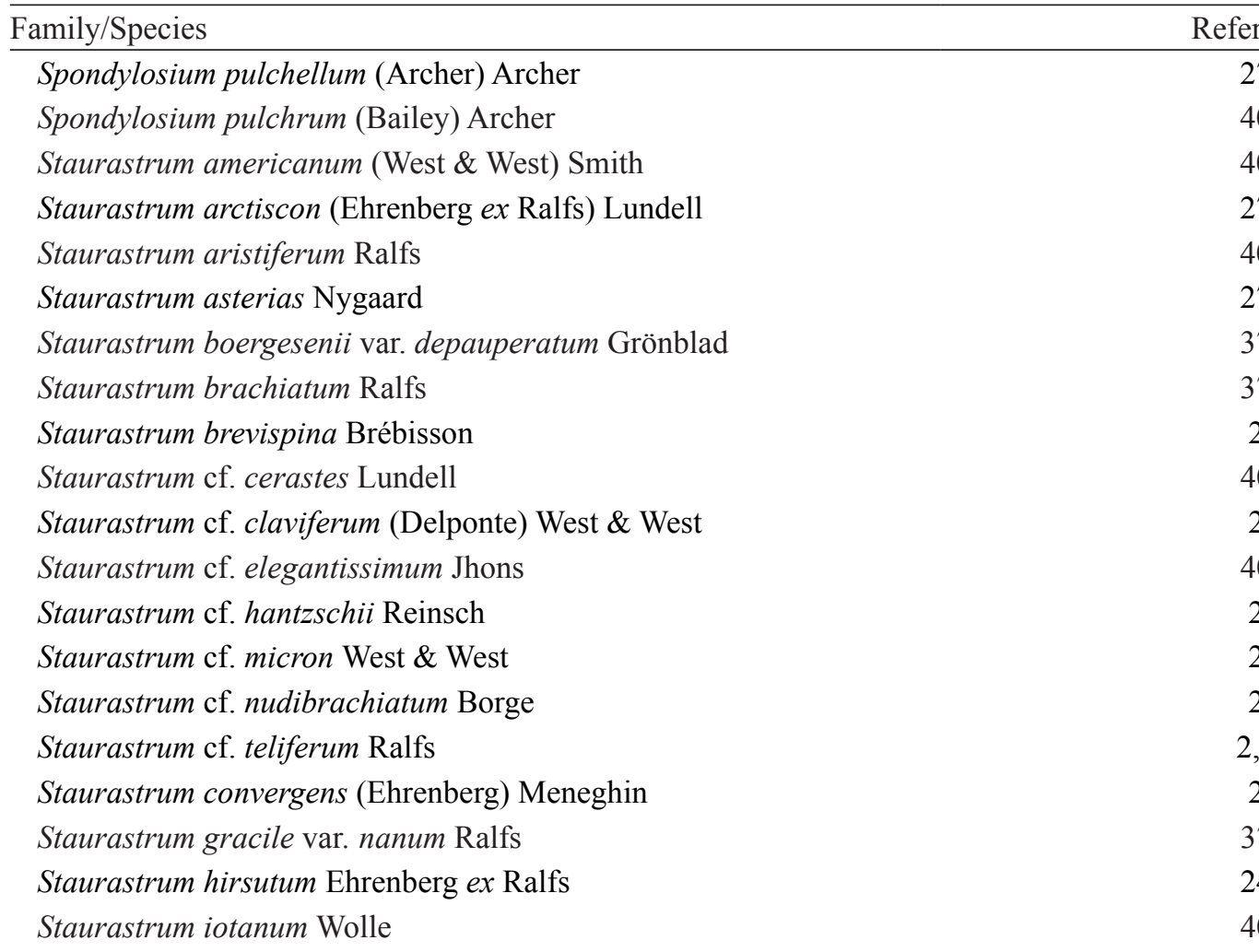

Staurastrum leptacanthum Nordstedt

$2,23,24,27$

Staurastrum leptacanthum Nordstedt var. borgei Förster

Staurastrum leptocladum var. borgei Förster

7

Staurastrum leptocladum var. cornutun Wille

Staurastrum leptocladum var. leptocladum Nordst

Staurastrum leptocladum var. subinsigne Scott et Grönblad 40

Staurastrum longipes (Nordstedt) 40

Staurastrum margaritaceum (Ehrenberg) ex Ralfs

$1,7,2,37^{*}$

Staurastrum muticum Brébisson ex Ralfs 27,40 *

Staurastrum nudibranchiatum Borge

$27,40 *$

Staurastrum pseudosebaldi Wille

Staurastrum quadrangulare Brébisson

Staurastrum quadrispinatum Turner

Staurastrum rotula Nordstedt

Staurastrum rugulosum var. angulare Grönblad

Staurastrum sebaldi Reinsch

Staurastrum setigerum Cleve

Staurastrum subpolymorphum Borge

Staurastrum tectum var. ayayense Grönblad

Staurastrum tetracerum (Kützing) Ralfs ex Ralfs

$27,28,40^{*}$

Staurastrum trifidum Nordstedt

$2,7,27,40^{*}$

Staurastrum volans West \& West

Staurodesmus clepsydra Nordstedt 
Table 2 (continuation)

\begin{tabular}{lc}
\hline Family/Species & Reference \\
\hline Staurodesmus convergens (Ehrenberg) Teiling & $2,40^{*}$ \\
Staurodesmus cuspidatus (Brébisson) Teiling & $7,27,40^{*}$ \\
Staurodesmus dejectus (Brébisson) Teiling & $23,27,28,40^{*}$ \\
Staurodesmus dickiei (Ralfs) Lillieroth & $7,23,24,27,40^{*}$ \\
Staurodesmus extensus (Andersson) Teiling & 40 \\
Staurodesmus glaber (Ehrenberg) Teiling & 37 \\
Staurodesmus glaber var. limnophilus Ehrenberg & 37 \\
Staurodesmus lobatus (Borge) Bourr & 40 \\
Staurodesmus mucronatus var. subtriangularis West \& West & 37 \\
Staurodesmus pachyrhynchus var. pachyrhynchus Nordstedt & 37 \\
Staurodesmus subulatus (Kützing) Croasd & 40 \\
Staurodesmus triangularis (Lagerheim) Teiling & 40 \\
Staurodesmus triangularis var. limneticus Teil & 37 \\
Staurodesmus validus (West \& West) Thom & 40 \\
Staurodesmus validus var. subvalidus Grönblad & 37 \\
Teilingia granulata (Roy \& Bisset) Bourrelly & 2,27 \\
Xanthidium antilopaeum var. mamillosum f. mediolaeve Grönblad & 37 \\
Xanthidium canadense (Joshua) Förster & 40 \\
Xanthidium concinum Archer & 37 \\
Xanthidium trilobum Nordstedt & $2,37 *$ \\
\hline
\end{tabular}

researchs each, and Aulacoseira distans (Ehrenberg) Simonsen and Dolichospermum spiroides (Klebahn) Wacklin, Hoffmann \& Komárek (Anabaena spiroides Klebahn) in five studies (table 2). In the total of taxa, 103 co-occurring to both habitats (table 2).

\section{Conclusion}

This study indicated that, since 1994 to 2016, 80 publications of some aspect of algae of the upper Paraná river floodplain were published. From these, 41 referenced algae at the generic and infrageneric level, and the compilation of them indicated 938 species, which constitutes an expressive microalgae richness for this study area. In the total, the Chlorophyceae class was the richest one followed by Zygnemaphyceae, but in the periphytic habitat Bacillariophyceae was the richest class, and in phytoplankton Chlorophyceae class. Some of these species may not be included in the Brazilian list of algae (which now has something around 4,747 species recorded so far) and now can be included.

This study contributed to the knowledge of the Brazilian microalgae biodiversity and provided data for future ecological and biogeographic studies.
This study also demonstrated the importance of long-term studies (PELD-CNPq site 6) in the survey of biodiversity. We suggest that these compilations be made also using research reports to increase information about the Brazilian flora and fauna, and improve the knowledge of Brazilian biodiversity.

\section{Acknowledgements}

We thank the Núcleo de Pesquisas em Limnologia, Ictiologia e Aquicultura from the Universidade Estadual de Maringá for supplying the infrastructure. $\mathrm{CNPq}$ and CAPES provided grants for researchers and CNPq/PELD for financial support.

\section{Literature cited}

Agostinho, A.A., Gomes, L.C., Thomaz, S.M. \& Hanh, N.S. 2004. The Upper Paraná River Floodplain: Main characteristics and perspectives for management and conservation. In: A.A. Agostinho (ed.). Conservação da biodiversidade em águas continentais do Brasil. Megadiversidade, pp. 70-78.

Algarte, V.M. \& Rodrigues, L. 2013. How periphytic algae respond to short-term emersion in a subtropical floodplain in Brazil. Phycologia 52: 557-564. 
Algarte, V.M., Moresco, C. \& Rodrigues, L. 2006. Algas do perifíton de distintos ambientes na planície de inundação do alto rio Paraná. Acta Scientiarum Biological Sciences 28: 243-251.

Algarte, V.M., Siqueira, N.S., Murakami, E.A. \& Rodrigues, L. 2009. Effects of hydrological regime and connectivity on the interannual variation in taxonomic similarity of periphytic algae. Brazilian Journal of Biology 69: 609-616.

Algarte, V.M., Rodrigues, L, Landeiro, V.L., Siqueira, T. \& Bini, L.M. 2014. Variance partitioning of deconstructed periphyton communities: does the use of biological traits matter? Hydrobiologia 722: 279-290.

Algarte, V.M., Dunck, B., Bichoff, A. \& Rodrigues, L. 2015. First record of Pithophora oedogonia (Montagne) Wittrock (Pithophoraceae) in the Upper Paraná River floodplain, Brazil. Check List 11: 1-4.

Algarte, V.M., Dunck, B., Leandrini, J.A. \& Rodrigues, L. 2016. Periphytic diatom ecological guilds in floodplain: Ten years after dam. Ecological Indicators 69: 407-414.

Bichoff, A., Osório, N.C., Dunck, B. \& Rodrigues, L. 2016. Periphytic algae in a floodplain lake and river under low water conditions. Biota Neotropica 16: e20160159.

Bicudo, C.E.M. \& Menezes, M. 2010. Introdução: As algas do Brasil. In: R. C. Forzza (ed.). Catálogo de plantas e fungos do Brasil. Instituto de Pesquisa Jardim Botânico do Rio de Janeiro, Rio de Janeiro, pp-49-60.

Biolo, S. \& Rodrigues, L. 2010. New records of Xanthophyceae and Euglenophyceae in the periphytic algal community from a Neotropical river floodplain, Brazil. Algological Studies 135:1-81.

Biolo, S. \& Rodrigues, L. 2011. Composição de algas perifíticas (exceto Bacillariophyceae) em distintos substratos naturais de um ambiente semilótico, planície de inundação do alto rio Paraná, Brasil. Revista Brasileira de Botânica 34: 307-319.

Biolo, S. \& Rodrigues, L. 2013A. Comparison of the structure of the periphytic community in distinct substrates from a Neotropical floodplain. International Research Journal of Plant Science 4: 64-75.

Biolo, S. \& Rodrigues, L. 2013B. Structure of the periphytic algae associated with a floating macrophyte in an open lake on the upper Paraná river floodplain, Brazil. Acta Scientiarum Biological Sciences 35: 415-419.

Biolo, S., Algarte, V.M. \& Rodrigues, L. 2015. Composition and taxonomic similarity of the periphytic algal community in different natural substrates in a neotropical floodplain, Brazil. African Journal of Plant Science 91: 17-24.

Bortolini, J.C., Bovo-Scomparin, V.M., De Paula, A.C.M., Moresco, G.A., Resi, L.M., Jati, S. \& Rodrigues, L.C. 2014. Composition and species richness phytoplankton in a subtropical floodplain lake: a long-term study. Acta Limnologica Brasiliensia 26: 296-305.
Bortolini, J.C., Train, S. \& Rodrigues, L.C. 2016. Extreme hydrological periods: effects on phytoplankton variability and persistence in a subtropical floodplain. Hydrobiologia 763: 223-236.

Bovo-Scomparin, V.M., Borges, P.A.F., Train, S. \& Rodrigues, L.C. 2005. Xanthophyceae planctônicas da planície de inundação do alto rio Paraná. Acta Scientiarum Biological Sciences 27: 9-20.

Bovo-Scomparin, V.M., Train, S. \& Rodrigues, L.C. 2013. Influence of reservoirs to dispersion and seasonal variation of the phytoplankton community in the Upper Paraná River, Brazil. Hydrobiolgia 702: 115-127.

Cabral, A. F., Dunck, B., Lansca-Toha, F.M., Rodrigues, L., Utz, L.R.P. \& Velho, L.F.M. 2014. First report of Colacium vesiculosum Ehrenberg 1853 (Euglenophyceae) as epibiont on planktonic copepods (Crustacea, Copepoda), in a Brazilian Floodplain lake. Acta Protozoologica 53: 335-340.

Dunck, B., Bortolini, Rodrigues, L.C., Rodrigues, L. Jati, S. \& Train, S. 2013. Functional diversity and adaptative strategies of planktonic and periphytic algae in isolated tropical floodplain lake. Brazilian Journal of Botany 36: 257-266.

Dunck, B., Rodrigues, L. \& Bicudo, D.C. 2015. Functional diversity and functional traits of periphytic algae during a short-term successional process in a Neotropical floodplain lake. Brazilian Journal of Biology 3: 1-10.

Dunck, B., Schneck, F. \& Rodrigues, L. 2016A. Patterns in species and functional dissimilarity: insights from periphytic algae in subtropical floodplain lakes. Hydrobiologia 1: 1-11.

Dunck, B., Algarte, V.M., Cianciaruso, M.V. \& Rodrigues, L. 2016B. Functional diversity and trait-environment relationships of periphytic algae in subtropical lakes. Ecological Indicators 67: 257-266.

Ferreira, A.F., Mormul, R.P., Biolo, S. \& Rodrigues, L. 2011. Podostemum rutifolium subsp. rutifolium como estruturador da comunidade de algas perifíticas em um rio neotropical. Rodriguésia 62: 813-825.

Fonseca, I.A. \& Rodrigues, L. 2004. Comunidade de algas perifíticas em distintos ambientes da planície de inundação do alto rio Paraná. Acta Scientiarum Biological Sciences 26: 1-7.

Fonseca, I.A. \& Rodrigues, L. 2005. Cianobactérias perifíticas em dois ambientes lênticos da planície de inundação do alto Rio Paraná, PR, Brasil. Revista Brasileira de Botânica 28: 821-834.

Fonseca, I.A. \& Rodrigues, L. 2007. Periphytic cyanobacteria in different environments from the upper Paraná river floodplain, Brazil. Acta Limnologica Brasiliensia 19: 53-65. 
Forzza, R.C., Baumgratz, J.F.A., Bicudo, C.E.M., Canhos, D.A.L., Carvalho Jr., A.A., Costa, A.F., Costa, D.P., Hopkins, M., Leitman, P.M., Lohmann L.G., Maia, L.C., Martinelli, G., Menezes, M., Morim, M.P., Nadruz-Coelho, M.A., Peixoto, A.L., Pirani, J.R., Prado, J., Queiroz, L.P., Souza, V.C., Stehmann, J.R., Sylvestre, L., Walter, B.M.T. \& Zappi, D. (eds.). 2010. Catálogo de plantas e fungos do Brasil. Andrea Jakobsson Estúdio/Jardim Botânico do Rio de Janeiro, Rio de Janeiro.

Grieneisen, M.L., Zhan, Y., Potter, D. \& Zhang, M. 2015. Biodiversity, taxonomic infrastructure, international collaboration, and new species discovery. BioScience 64: $322-332$

Jati, S. \& Train, S. 1993. Representantes do gênero Trachelomonas Ehrenberg, de duas lagoas da ilha Porto Rico, município de Porto Rico, Paraná, Brasil. Revista Unimar 15: 37-51.

Jati, S. \& Train, S. 1994. Euglenophycea pigmentadas de ambientes lênticos da Ilha Porto Rico, Município de Porto Rico, Paraná, Brasil. Iheringia, Serie Botânica 45: 117-142.

Menezes, M., Bicudo, C.E.M. \& Moura, C.W.N. 2015. Update of the Brazilian floristic list of Algae and Cyanobacteria. Rodriguésia 66: 1047-1062.

Moresco, G.A., Paula, A.C.M., Bortolini, J.C., Jati, S., Reis, L.M. \& Rodrigues, L.C. 2015. Zygnemaphyceae em um lago de várzea na planície de inundação do alto rio Paraná: Gêneros Closterium, Cosmarium, Euastrum, Micrasterias e Pleurotaenium. Iheringia Serie Botânica 70: 143-155.

Mormul, R.P., Thomaz, S.M., Silveira, M.J. \& Rodrigues, L. 2010. Epiphyton or Macrophyte: Which Primary Producer Attracts the Snail Hebetancylus moricandi? American Malacological Bulletin 28: 127-133.

Murakami, E.A. \& Rodrigues, L. 2009. Resposta das algas perifíticas às alterações de temperatura e ao enriquecimento artificial de nutrientes em curto período de tempo. Acta Scientiarum Biological Sciences 31: 273-284.

Neif, E.M., Behrend, R.D.L. \& Rodrigues, L. 2013. Seasonal dynamics of the structure of epiphytic algal community on different substrates from a Neotropical floodplain. Revista Brasileira de Botânica 1: 1-11.

Neif, E.M., Behrend, R.D.L. \& Rodrigues, L. 2014. Investigations on periphytic algae: comparing distinct years in the presence and absence of submerged macrophytes. Brazilian Journal of Biology 74: 521-522.

Oliveira, M.D., Jati, S. \& Train, S. 1994. Levantamento preliminar de fitoplancton de rede (exceto zignemaphyceae) do rio Paraná, no município de Porto Rico, Paraná, Brasil. Unimar 16: 155-174.
Osório, N.C., Tremarin, P.I., Ludwig, T.V. \& Rodrigues, L. 2016. Gomphonema Ehrenberg (Bacillariophyceae) in a lotic environment of the Upper Paraná River floodplain, Brazil. Acta Scientiarum Biological Sciences online first.

Paula, A.C.M., Moresco, G.A., Bortolini, J.C., Jati, S., Reis, L.M. \& Rodrigues, L.C. 2014. Os gêneros Staurastrum, Staurodesmus e Xanthidium em uma lagoa de inundação, planície de inundação do alto rio Paraná, Brasil. Iheringia Serie Botânica 69: 417-431.

Rodrigues, L. \& Bicudo, D.C. 2004. Periphytic Algae. In: S.M. Thomaz (ed.). The Upper Paraná River and its Floodplain: physical aspects, ecology and conservation Leiden Backhuys Publishers, pp. 125-143.

Rodrigues, L. \& Bicudo, D.C. 2001. Similarity among periphyton algal communities in a lentic-lotic gradient of the upper Paraná river floodplain, Brazil. Revista Brasileira de Botânica 24: 235-248.

Rodrigues, L.C., Train, S., Bovo-Scomparin, V.M., Jati, S., Borsalli, C.C.J. \& Maregoni, E. 2009. Interannual variability of phytoplankton in the main rivers of the Upper Paraná River floodplain, Brazil: influence of upstream reservoirs. Brazilian Journal of Biology 69: 501-516.

Round, F.E. 1971. The biology of algae. London: Edward Arnold (Publishers) Ltd.

Takemoto, R.M. \& Lizama, M.L.A.P. 2010. Helminth fauna of fishes from the upper Paraná rive floodplain, Brazil. Neotropical Helminthology 4: 5-8

Train, S. \& Rodrigues, L.C. 1998. Temporal fluctuations of the phytoplankton community of the Baía River, in the upper Paraná River floodplain, Mato Grosso do Sul, Brazil. Hydrobiologia 361: 125-134.

Train, S., Oliveira, M.D. \& Quevedo, M.T. 2000. Dinâmica sazonal da comunidade fitoplanctônica de um canal lateral (Canal Cortado) do Alto Rio Paraná (PR, Brasil). Acta Scientiarum Biological Science 22: 389-395.

Train, S., Rodrigues, L.C., Bovo-Scomparin, V.M., Borges, P.A.F. \& Pivato, B.M. 2004. Phytoplankton Composition and Biomass in environments of the Upper Paraná River Floodplain. In: A.A. Agostinho (ed.). Structure and functioning of the Paraná River and is floodplain, LTER- site 6. Eduem, Maringá, pp. 233-238.

Train, S. \& Rodrigues, L.C. 2004. Phytoplankton assemblages. In: S.M. Thomaz, A.A. Agostinho \& N.S. Hahn (eds.). The Upper Paraná river floodplain: physical aspects, ecology and conservation. Backhuys, Netherlands, pp. 103-124. 